THE ASTROPHYSICAL JOURNAL, 332:995-1008, 1988 September 15

(C) 1988. The American Astronomical Society. All rights reserved. Printed in U.S.A.

\title{
THE SMALL MOLECULAR CLOUD TOWARD HD 169454
}

\author{
Buell T. Jannuzi, John H. Black, AND Charles J. Lada \\ Steward Observatory
}

AND

EWINE F. VAN DishoecK

Princeton University Observatory

Received 1987 December 18; accepted 1988 March 3

\begin{abstract}
Optical absorption line observations of the B1 supergiant HD 169454 reveal the presence of an intervening translucent interstellar cloud. Millimeter wavelength observations of $\mathrm{CO}$ emission show that the absorption lines can be attributed to a well-defined cloud approximately $18^{\prime}$ by $22^{\prime}$ in extent at the same radial velocity as the $\mathrm{C}_{2}$ and $\mathrm{CN}$ absorption lines. The map of the $\mathrm{CO}$ emission shows three large condensations and evidence of unresolved structure on smaller scales. The measured rotational excitation of the $\mathrm{C}_{2}$ implies a kinetic temperature $T=15_{-5}^{+10} \mathrm{~K}$ and a density $n\left(\mathrm{H}_{2}\right) \approx 300 \mathrm{~cm}^{-3}$. Various procedures for determining the cloud mass are compared. The estimated mass is $7-14 M_{\odot}$ if the cloud lies at the distance $(D \approx 125 \mathrm{pc})$ of an extended atomic cloud at the same radial velocity and is $250-500 M_{\odot}$ if the kinematic distance $(D \approx 750 \mathrm{pc})$ is adopted. The dynamical state of the cloud is examined. The composition and structure of the cloud are discussed with reference to detailed theoretical models and the properties of other interstellar clouds.
\end{abstract}

Subject headings: interstellar: molecules — stars: individual (HD 169454)

\section{INTRODUCTION}

Sensitive detectors with high-resolution spectrographs make possible the observation of interstellar absorption lines in translucent regions intermediate between the classical diffuse and the thick opaque interstellar clouds. At the same time, continuing development of sensitive millimeter receivers and the availability of millimeter telescopes allow astronomers to study the weaker emission of molecular gas from clouds smaller and less massive than giant molecular clouds (GMCs) or dark clouds. Three recent surveys for $\mathrm{CO}$ emission at high Galactic latitude have found significant amounts of molecular gas. The median inferred masses for the detected clouds in these surveys are $18 M_{\odot}$ (Blitz, Magnani, and Mundy 1984; Magnani, Blitz, and Mundy 1985), $2 M_{\odot}$ (Keto and Myers 1986), and $6 M_{\odot}$ (de Vries, Heithausen, and Thaddeus 1987). Although individually each of the observed clouds is small, collectively they might constitute as much as $10 \%$ of the molecular gas in the Galaxy (Magnani, Lada, and Blitz 1986). More recently, Knapp and Bowers (1987) have reported the discovery of several "tiny molecular clouds" in the direction of $\alpha$ Ori, whose masses are estimated to be of the order of $1 M_{\odot}$ or less. Current knowledge of the distribution and overall importance of such clouds is primitive since large-scale emission line surveys for diffuse and translucent molecular clouds have not yet been attempted. It is possible that a significant fraction of molecular gas in the Galaxy is in a more dilute component than the GMCs.

While surveys are important for understanding the extent and Galactic distribution of small molecular clouds, the methods used to infer individual cloud masses and physical conditions from survey data must be tested carefully. This can be accomplished by detailed study of individual clouds. The small molecular cloud associated with the Pleiades has received much attention. Its discovery has been attributed to R. S. Cohen, whose unpublished work in 1981 has been used extensively by Gordon and Arny (1984), Federman and Willson (1984), Breger (1986, 1987), and Guthrie (1984). Additional recent studies of the molecular material in the Pleiades region include those of White $(1984 a, b)$. The distance to this cloud is well determined and its mass is estimated to be $20 M_{\odot}$ on the basis of measured interstellar extinction.

As pointed out in studies of the Taurus (Crutcher 1985) and Ophiuchus (Crutcher and Chu 1985) molecular clouds, the use of both optical and radio observations has several advantages over studies based on radio observations alone. Radio observations of ${ }^{12} \mathrm{CO}$ and ${ }^{13} \mathrm{CO}$ emission provide direct measurements of the size, morphology, and kinematics of the $\mathrm{CO}$ emitting component of the cloud. They also allow indirect estimates of the total column density of $\mathrm{CO}, N(\mathrm{CO})$, total column density of molecular hydrogen, $N\left(\mathrm{H}_{2}\right)$, distance to the cloud, and mass of the cloud. Unfortunately, radio observations are limited in their angular resolution. If a background star suitable for high-resolution interstellar line spectroscopy is present, optical observations can provide constraints on the distance to the star, measurements of some molecular column densities ( $\mathrm{CH}, \mathrm{CN}, \mathrm{C}_{2}$ for example), and a measure of extinction. The $\mathrm{C}_{2}$ molecule, in particular, affords diagnostic probes of temperature, density, and intensity of starlight (van Dishoeck and Black 1982). The cloud toward the B1 supergiant HD 169454 provides a particularly valuable test case of the methods used to derive physical properties of small molecular clouds because of the availability of complementary optical and radio data. We present an initial investigation of the structure and physical state of this cloud. The observations are summarized in $\S$ II. In $\S$ III, we discuss various observed and derived cloud properties. The derived properties (including density, kinetic temperature, and cloud mass) presented in $\S$ III are based on simple arguments and simplifying assumptions. In $\S$ IV, a detailed model of the cloud is presented and compared to the observations. 


\section{OBSERVATIONS}

Millimeter observations were made by B. T. J. with the $5 \mathrm{~m}$ telescope of the Millimeter Wave Observatory of the University of Texas at Austin (MWO) ${ }^{1}$ and the NRAO $12 \mathrm{~m}$ telescope on Kitt Peak. ${ }^{2}$ All observations at both telescopes were made in position switching mode. Absorption line data were obtained by E. F. vD. and J. H. B. at several telescopes as part of optical surveys of diffuse clouds. The observing procedure and reduction of the data are discussed below.

\section{a) Millimeter Wave Observations}

Observations were made of the $J=1 \rightarrow 0$ emission of ${ }^{12} \mathrm{C}^{16} \mathrm{O}(115.271 \mathrm{GHz})$ from an extended region in the direction of HD $169454\left(\alpha_{1950}=18^{\mathrm{h}} 22^{\mathrm{m}} 24^{\mathrm{s}} 89, \delta_{1950}=-14^{\circ} 0^{\prime} 25^{\prime \prime} .5\right.$; $l=17.53, b=-0.670$ ), during the period 1985 June $20-27$ at the MWO. At $115 \mathrm{GHz}$ the beam of the $5 \mathrm{~m}$ is approximately 2.3 arcminutes (HPBW $=2.3$ ). The $250 \mathrm{kHz}$ filter banks provided a channel width of $0.65 \mathrm{~km} \mathrm{~s}^{-1}$. System temperatures ranged from 800 to $1200 \mathrm{~K}$. Standard sources were regularly observed. Calibration was done using the ambient temperature chopper wheel method described in Ulich and Haas (1976) and Penzias and Burrus (1973). Line temperatures are presented in terms of $T_{R}^{*}$ : i.e., the source antenna temperature corrected for atmospheric, ohmic, and all spillover losses. For the MWO data, the correction for forward scattering and spillover is $\eta_{\text {fss }}=0.86$ (Kutner and Ulich 1981). Note that $T_{R}^{*}$ is related to the true Rayleigh-Jeans radiation temperature, $T_{R}$, by a correction for the coupling efficiency of the antenna beam to the brightness distribution of the source $\left(T_{R}^{*}=\eta_{c} T_{R}\right)$. No correction has been applied for this effect, but for extended sources $\eta_{c}$ is usually very close to one. Calibration errors are estimated to be $15 \%$ to $20 \%$.

Observations of selected regions of the cloud were made using the NRAO $12 \mathrm{~m}$ telescope. Observations of the ${ }^{12} \mathrm{C}^{16} \mathrm{O}$

${ }^{1}$ The MWO is operated by the Electrical Engineering Research Laboratory of the University of Texas at Austin with support from the NSF and McDonald Observatory.

${ }^{2}$ The National Radio Astronomy Observatory is operated by Associated Universities, Inc., under contract with the National Science Foundation. and ${ }^{13} \mathrm{C}^{16} \mathrm{O}(110.201 \mathrm{GHz}) J=1 \rightarrow 0$ emission were made 1985 December 5-8. At $2.6 \mathrm{~mm}$ the diameter of the beam of the $12 \mathrm{~m}$ is 1 minute of arc $\left(\mathrm{HPBW}=1^{\prime}\right)$. A single $30 \mathrm{kHz}$ and two $100 \mathrm{kHz}$ filter banks were used simultaneously providing 0.078 $\mathrm{km} \mathrm{s}^{-1}$ and $0.26 \mathrm{~km} \mathrm{~s}^{-1}$ resolution, respectively. The two 100 $\mathrm{kHz}$ filter banks were used in parallel for increased sensitivity. The system temperature ranged from $600 \mathrm{~K}$ to $1100 \mathrm{~K}$. Calibration was performed by the chopper wheel method, with adoption of standard Kitt Peak values for the calibration temperature as a function of air mass at $115 \mathrm{GHz}$. The temperature scale was checked by observation of standard sources. At 110 $\mathrm{GHz}$ a constant calibration temperature $T_{C}=800 \mathrm{~K}$ was used, and the corrections for air mass and changing ambient temperature were made during reduction of the data following the procedure of Ulich and Haas (1976). This was done because of uncertainty about the temperature and airmass corrections available during real time observations and because of the lack of a suitable ${ }^{13} \mathrm{CO} J=1 \rightarrow 0$ emission calibration source. Errors in the calibration are estimated to be $15 \%$ to $20 \%$.

In what follows, all position offsets in arcminutes $(\Delta \alpha, \Delta \delta)$, refer to the star HD 169454 at $(\Delta \alpha, \Delta \delta)=(0,0)$. Table 1 summarizes the $\mathrm{CO}$ measurements at three positions in the cloud: $(\Delta \alpha, \Delta \delta)=(0,0),(0,2)$, and $(-2,2)$. The latter two positions are the locations of highest antenna temperature in the MWO and NRAO maps. The $J=2 \rightarrow 1$ data of Lada and Blitz (1987) have been included in the table for comparison. The tabulated line parameters are determined from fits of single Gaussian functions to the line profiles: these are the peak temperature $T_{R}^{*}$, the full width at half-maximum of the profile $\Delta V$, and the line center radial velocity $V_{\mathrm{LSR}}$. The integrated line intensity $I=\int T_{R}^{*}(V) d V$ is determined by direct integration over the observed line profile, not from the fitted parameters. Radial velocities of the optical absorption lines are also listed.

\section{b) Absorption Line Observations}

Data on the interstellar $\mathrm{C}_{2} A^{1} \Pi_{u}-X{ }^{1} \Sigma_{g}^{+}(2,0)$ lines $(\lambda \approx 8750 \AA)$ and CN $A^{2} \Pi-X^{2} \Sigma^{+}(2,0)$ lines $(\lambda \approx 7900 \AA)$ in the spectrum of HD 169454 were obtained by E. F. vD. with the $1.4 \mathrm{~m}$ coudé auxiliary telescope and coudé echelle spectrometer at the European Southern Observatory 1984 August

TABLE 1

Selected Observational Data

\begin{tabular}{|c|c|c|c|c|c|c|c|}
\hline Species & Transition & $\begin{array}{l}(\Delta \alpha, \Delta \delta) \\
(\operatorname{arcmin})\end{array}$ & $\begin{array}{r}T_{R}^{*} \\
(\mathrm{~K})\end{array}$ & $\begin{array}{c}\Delta V^{\mathrm{a}} \\
\left(\mathrm{km} \mathrm{s} \mathrm{s}^{-1}\right)\end{array}$ & 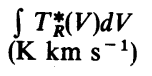 & $\begin{array}{c}V_{\mathrm{LSR}} \\
\left(\mathrm{km} \mathrm{s}^{-1}\right)\end{array}$ & Telescope \\
\hline${ }^{12} \mathrm{C}^{16} \mathrm{O}$ & $J=1 \rightarrow 0$ & $(0,0)$ & $4.4 \pm 0.2$ & $1.3 \pm 0.5$ & $6.1 \pm 0.4$ & $5.9 \pm 0.3$ & MWO \\
\hline${ }^{12} \mathrm{C}^{16} \mathrm{O}$ & $J=1 \rightarrow 0$ & $(0,0)$ & $4.7 \pm 0.1$ & $1.3 \pm 0.1$ & $6.5 \pm 0.4$ & $6.10 \pm 0.1$ & NRAO \\
\hline${ }^{13} \mathrm{C}^{16} \mathrm{O}$ & $J=1 \rightarrow 0$ & $(0,0)$ & $1.20 \pm 0.09$ & $0.76 \pm 0.08$ & $1.0 \pm 0.3$ & $5.87 \pm 0.1$ & NRAO \\
\hline${ }^{12} \mathrm{C}^{16} \mathrm{O}$. & $J=2 \rightarrow 1$ & $(0,0)$ & $3.5 \pm 0.3$ & $\overline{1.4}$ & 5.2 & 5.9 & $\mathbf{M W O}^{\mathbf{b}}$ \\
\hline${ }^{13} \mathrm{C}^{16} \mathrm{O} \ldots$ & $J=2 \rightarrow 1$ & $(0,0)$ & $0.7 \pm 0.2$ & 0.6 & 0.45 & 5.8 & $\mathbf{M W O}^{\mathrm{b}}$ \\
\hline${ }^{12} \mathrm{C}^{16} \mathrm{O} \ldots \ldots \ldots \ldots$ & $J=1 \rightarrow 0$ & $(0,2)$ & $5.1 \pm 0.2$ & $1.1 \pm 0.5$ & $6.0 \pm 0.3$ & $5.9 \pm 0.2$ & MWO \\
\hline${ }^{12} \mathrm{C}^{16} \mathrm{O} \ldots \ldots \ldots \ldots$ & $J=1 \rightarrow 0$ & $(0,2)$ & $4.9 \pm 0.2$ & $1.3 \pm 0.1$ & $6.7 \pm 0.8$ & $5.88 \pm 0.1$ & NRAO \\
\hline${ }_{12}^{13} \mathrm{C}^{16} \mathrm{O} \ldots \ldots \ldots \ldots$ & $J=1 \rightarrow 0$ & $(0,2)$ & $1.53 \pm 0.06$ & $0.60 \pm 0.1$ & $0.98 \pm 0.27$ & $5.92 \pm 0.1$ & NRAO \\
\hline${ }_{12}^{12} \mathrm{C}^{16} \mathrm{O} \ldots$ & $J=1 \rightarrow 0$ & $(-2,2)$ & $4.6 \pm 0.2$ & $1.0 \pm 0.5$ & $4.9 \pm 0.3$ & $5.9 \pm 0.2$ & MWO \\
\hline $\begin{array}{l}{ }^{12} \mathrm{C}^{16} \mathrm{O} \ldots \\
{ }^{13} \mathrm{C}^{16} \mathrm{O}\end{array}$ & $J=1 \rightarrow 0$ & $(-2,2)$ & $6.3 \pm 0.4$ & $0.9 \pm 0.1$ & $5.9 \pm 1.3$ & $5.97 \pm 0.1$ & NRAO \\
\hline${ }^{13} \mathrm{C}^{16} \mathrm{O} \ldots \ldots \ldots \ldots$ & $J=1 \rightarrow 0$ & $(-2,2)$ & $2.1 \pm 0.2$ & $0.71 \pm 0.08$ & $1.6 \pm 0.6$ & $5.94 \pm 0.1$ & NRAO \\
\hline $\mathrm{C}_{2} \quad \cdots \cdots \cdots \cdots \cdots \cdots$ & $A-X(2,0)$ & $(0,0)$ & .. & $\ldots$ & $\ldots$ & $7.1 \pm 1.5$ & ESO \\
\hline$\cdots \cdots+\cdots, \cdots, \cdots$ & $A-X(2,0)$ & $(0,0)$ & $\cdots$ & $\ldots$ & $\ldots$ & 5.8 & $\mathrm{ESO}^{\mathrm{c}}$ \\
\hline $\mathrm{CN} \ldots \ldots \ldots \ldots \ldots$ & $A-X(2,0)$ & $(0,0)$ & $\ldots$ & $\ldots$ & $\ldots$ & $4.3 \pm 2$ & ESO \\
\hline $\mathrm{CH}, \ldots \ldots \ldots \ldots \ldots$ & $A-X(0,0)$ & $(0,0)$ & $\ldots$ & $\ldots$ & $\ldots$ & $3.6 \pm 2.4$ & MMT \\
\hline
\end{tabular}

a Line widths for NRAO measurements are based on the $30 \mathrm{KHz}$ filter bank data for which the effects of instrumental broadening are negligible.

b Data from Lada and Blitz 1987.

c Data from Gredel and Münch 1986. 
21-28. The resolution of these spectra was $3.75 \mathrm{~km} \mathrm{~s}^{-1}$. Spectra of the CH $A^{2} \Delta-X^{2} \Pi(0,0) R_{f}(1 / 2)$ feature $(\lambda=4300.3132 \AA)$ were obtained by J. H. B. with the echelle spectrograph at the Multiple Mirror Telescope, 1986 May 23-25, at a resolution of approximately $12.5 \mathrm{~km} \mathrm{~s}^{-1}$ Details of these observations and their interpretation will be presented elsewhere (van Dishoeck and Black 1988a). A preliminary report of the $\mathrm{C}_{2}$ and $\mathrm{CN}$ measurements has appeared (van Dishoeck and Black 1984) and the $\mathrm{C}_{2}$ has been observed independently with the same instrument by Gredel and Münch (1986).

\section{CLOUD PROPERTIES}

\section{a) Distance to the Cloud}

The interstellar absorption lines of $\mathrm{CH}, \mathrm{C}_{2}$ and $\mathrm{CN}$ arise in a single velocity component at a radial velocity, $V_{\mathrm{LSR}} \approx+6 \mathrm{~km}$ $\mathrm{s}^{-1}$ with respect to the local standard of rest. A remarkable feature of the $\mathrm{CO}$ emission in the direction of HD 169454 is that the $V_{\mathrm{LSR}} \approx+6 \mathrm{~km} \mathrm{~s}^{-1}$ cloud is quite distinct from background emission, despite the low Galactic latitude, $b=$ -0.67. The star HD 169454 (V430 Sct) was listed by Humphreys (1978) as one of the more luminous stars in the Galaxy, with a classification $\mathrm{B} 1 \mathrm{Ia}^{+}$and an absolute visual magnitude $M_{V}=-8$. She assigned it membership in the Sct OB3 association for which the mean distance is $1660 \mathrm{pc}$. Seab, Snow, and Joseph (1981) have noted that the extinction curve for this star is abnormal in the ultraviolet; however, under the assumption of a conventional value for the ratio of total to selective extinction, $A_{V} / E(B-V)=3.1$, the observed color excess, $E(B-V)=1.14$, would imply a visual extinction $A_{V}=3.53$ mag. Adoption of an absolute magnitude appropriate for a less luminous Ia supergiant, $M_{V}=-7$ (Walborn 1972), would place the star at a distance $D_{*}=1000 \mathrm{pc}$. Absolute magnitudes as bright as $M_{V}=-8.5$ have been suggested, in which case $D_{*}=2100 \mathrm{pc}$ (Sterken and Wolf 1978). We adopt the association distance, $1660 \mathrm{pc}$, as the best estimate for the star and take $2100 \mathrm{pc}$ to be an extreme upper limit. The stellar radial velocity is poorly determined owing to the large range of velocities in the extended atmosphere and their variability (Sterken and Wolf 1978), nor is there any other evidence that the $V_{\mathrm{LSR}}=$ $+6 \mathrm{~km} \mathrm{~s}^{-1}$ cloud is physically associated with the star. In any case, the cloud must lie in the foreground. The mean line center velocity of the ${ }^{12} \mathrm{CO}$ emission in the cloud is $V_{\mathrm{LSR}}=+5.6 \mathrm{~km}$ $\mathrm{s}^{-1}$. The kinematic distance for this velocity in the Galactic rotation model of Gunn, Knapp, and Tremaine (1979) is $D=680 \mathrm{pc}$, a value consistent with the stellar distance (i.e., $D<D_{*}$ ). Such kinematic distance estimates are subject to large uncertainties; for example, within $2 \mathrm{kpc}$ deviations of $\pm 5 \mathrm{~km}$ $\mathrm{s}^{-1}$ are observed in CO emitting clouds (Clemens 1985), corresponding to $\pm 500 \mathrm{pc}$ in the kinematic distance.

R. Crutcher has called to our attention the fact that the position and radial velocity of the cloud suggest an association with a large, nearby cloud of cold atomic hydrogen first surveyed by Riegel and Crutcher (1972) as a prominent selfabsorption feature in the $21 \mathrm{~cm}$ line. The distance to the cold atomic cloud at $V_{\mathrm{LSR}}=+4$ to $+7 \mathrm{~km} \mathrm{~s}^{-1}$ is well constrained by optical absorption line observations of 49 stars to be $D=125 \pm 25$ pc (Crutcher and Lien 1984). Our molecular cloud does not show up as a noticeable peak in the $21 \mathrm{~cm}$ self-absorption; however, its full angular extent is just comparable to the angular resolution $\left(22^{\prime}\right)$ of the measurements of Riegel and Crutcher (1972). Moreover, the H I need not peak on a molecular cloud. As will be shown below, the measured
H I column density is comparable to that expected for such a molecular cloud.

Although the agreement in velocity and position with an extremity of the nearby, cold $\mathrm{H}$ I cloud is highly suggestive of a physical association, the distance to the molecular cloud still needs to be determined unambiguously. The method of Crutcher and Lien (1984) could also be applied to the molecular cloud if enough early-type stars at $D=50-300 \mathrm{pc}$ could be identified and studied spectroscopically. An A0 V star at $D<150$ pc with $A_{V} \leq 3.5$ mag would be at least as bright as $m_{V} \approx 10$ mag. We will discuss interpretations based on the two distance estimates separately. All distance-dependent quantities will be identified.

\section{b) Spatial Morphology: Size and Clumping}

The observations made at MWO provide one of the first maps of the full extent of the ${ }^{12} \mathrm{CO} J=1 \rightarrow 0$ emission from a small molecular cloud in the galactic plane. Figure 1 is a map made from data obtained at MWO of the integrated intensity $\left(\int T_{R}^{*} d V\right)$ of the ${ }^{12} \mathrm{CO}$ emission $(J=1 \rightarrow 0)$. Figure 2 is a map of the peak $T_{R}^{*}$ observed at each pointed observation. The cloud is well-defined spatially and has been completely mapped at $2^{\prime}$ intervals to the $1 \mathrm{~K}$ contour in peak $T_{R}^{*}$. While clearly isolated from background $\mathrm{CO}$ emission, the small molecular cloud toward HD $169454(l=17.5, b=-0.67)$ has a neighbor with the same velocity, $V_{\mathrm{LSR}}=+6$, located at $l=$ $18.3, b=-0.3$. The Massachusetts-Stony Brook Galactic plane CO survey shows both of these cloud features (Sanders et al. 1986; Clemens et al. 1986). The two clouds are clearly distinct at the level of the noise in the survey data where $3 \sigma$ corresponds to $1.25 \mathrm{~K}$. In a map of integrated $J=1 \rightarrow 0 \mathrm{CO}$ emission at $V_{\mathrm{LSR}}=5.5-6.5 \mathrm{~km} \mathrm{~s}^{-1}$, the $1 \mathrm{~K}$ contours of the two clouds are separated by $13^{\prime}$. This does not eliminate the possibility that weak emission $(<1 \mathrm{~K})$ might be detectable surrounding and connecting the two clouds. The angular extent of the ${ }^{12} \mathrm{CO}$ emission from the small cloud is approximately $20^{\prime}$ by $18^{\prime}$ and covers 330 square minutes of arc. If we assume that the cloud is roughly spherical in shape, it has a diameter $d=4.4$ (or 0.73 ) pc, at a distance $D=750$ (or 125) pc. In making this calculation we have defined the angular size to be the angular diameter of the $1 \mathrm{~K}$ contour of the ${ }^{12} \mathrm{CO}$ emission peak $T_{R}^{*}$ map. Martin and Barrett (1978) use a similar definition in their work on dark globules.

Examination of Figures 1 and 2 shows three regions of localized emission. These regions are labeled $\mathrm{A}, \mathrm{B}$, and $\mathrm{C}$ (from north to south). In these maps the three regions or "clumps" appear to be separated spatially and connected by weaker emission. We will see below that the line center velocities of each clump are distinct. While the cloud (all of the observed emission within the continuous $1 \mathrm{~K}$ peak $T_{R}^{*}$ map) has a diameter of about $20^{\prime}$, these three "clumps" range from $6^{\prime}$ to $10^{\prime}$ in diameter. These maps were made with a 2.3 beam, which allows us to resolve structures approximately 0.5 (or 0.08 ) pc in size at a distance $D=750$ (or 125) pc. If we now compare the appropriate regions of Figures 1 and 2 with the maps of "clump" B made from the $12 \mathrm{~m}$ data (Figs. 3 and 4), we see that there is structure on even smaller scales. What appeared as a single "clump" is now resolvable into at least two clumps. The higher resolution of the $12 \mathrm{~m}$ reveals structure on the scale of 0.2 (or 0.04 ) pc at $D=750$ (or 125 ) pc. Even with the $1^{\prime}$ beam there is still evidence of unresolved structure. From these maps, we cannot set a limit on the smallest scale of "clumping" that might exist in the cloud. The evidence for small-scale clumping 


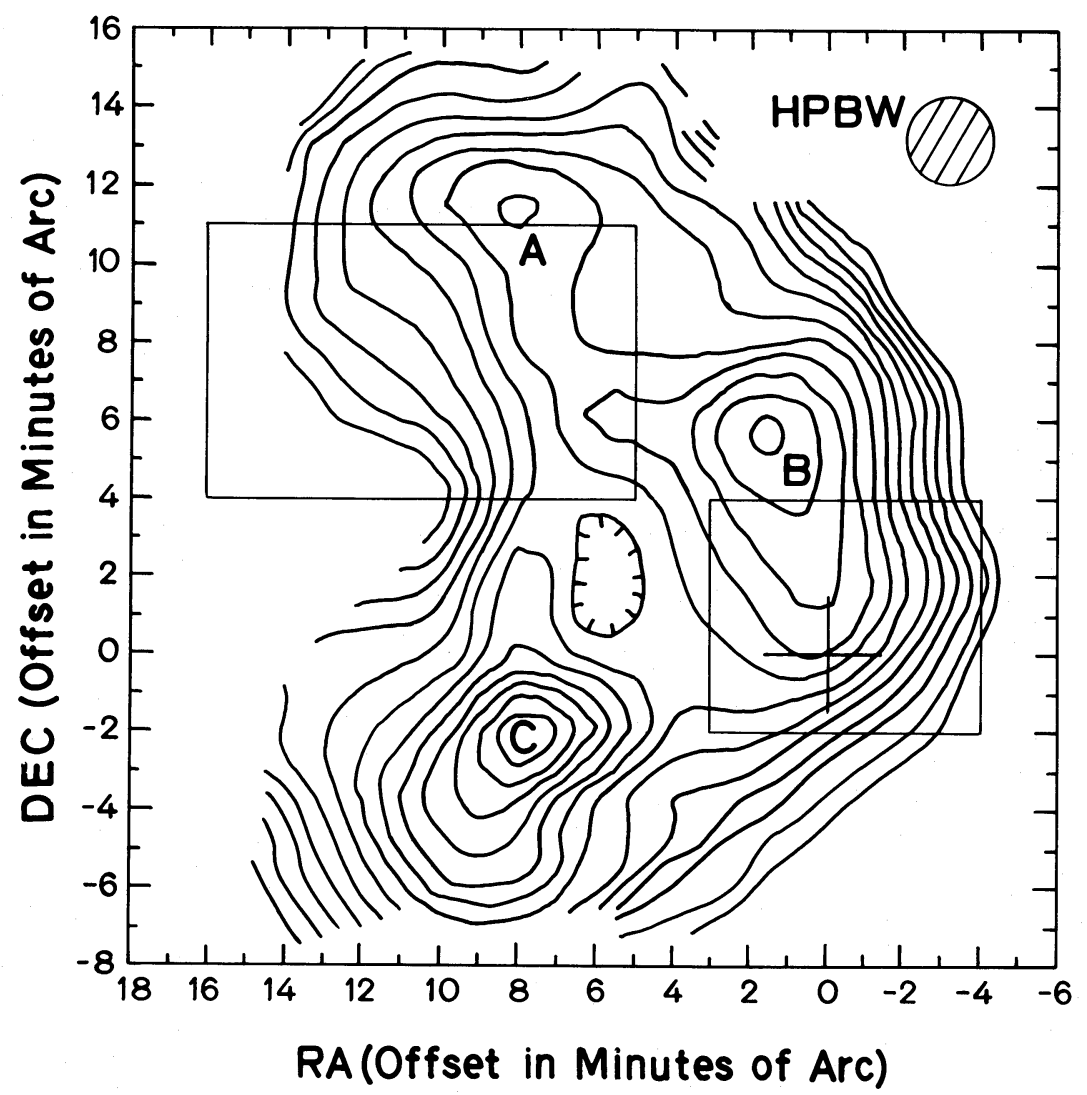

FIG. 1.- Map of the integrated intensity $\left(\int T_{R}^{*} d V\right)$ of ${ }^{12} \mathrm{CO} J=1 \rightarrow 0$ emission from the molecular cloud toward HD 169454. This map is made from the data obtained with the MWO $5 \mathrm{~m}$ telescope. The telescope beam (HPBW) is $2^{\prime} .3$. Observations were spaced at $2^{\prime}$ intervals. Open contours indicate holes in the coverage of the observations. The plus sign indicates the position of $\mathrm{HD} 169454\left(\alpha_{1950}=18^{\mathrm{h}} 22^{\mathrm{m}} 24^{\mathrm{s}} 89, \delta_{1950}=-14^{\circ} 0^{\prime} 25^{\prime \prime} .5\right)$. The letters A, B, and C on the map indicate three regions of emission referred to in the text as "clumps." The lowest contour is $1.0 \mathrm{~K} \mathrm{~km} \mathrm{~s}^{-1}$. Each additional contour indicates an increase of $0.5 \mathrm{~K} \mathrm{~km} \mathrm{~s}$. The axes display the offset in arcmin from the position of the star HD 169454. The box indicates the region mapped at higher angular resolution with the NRAO $12 \mathrm{~m}$ telescope.

and its effects on the internal structure and evolution of molecular clouds have been reviewed recently by Falgarone and Pérault (1987) and Blitz (1987). In considering all derived properties of such clouds it is important to keep in mind that the generally assumed uniformity in density and symmetry does not actually exist, and that this could affect the accuracy of some derived properties.

\section{c) Velocity Structure of the Cloud}

We have seen that with increasing spatial resolution there is structure on increasingly smaller scales. Our maps of the CO emission display the projected distribution of the molecular gas. We can also examine the velocity structure of the emission for further clues to the morphology and dynamics of the cloud.

The observed ${ }^{12} \mathrm{CO}$ line profiles obtained with the MWO $5 \mathrm{~m}$ telescope have been fitted with single Gaussians. The widths of the ${ }^{12} \mathrm{CO}$ emission lines at each observed position are narrow, ranging from $\Delta V=0.6$ to $2.1 \mathrm{~km} \mathrm{~s}^{-1}$, with a mean value of $1.2 \mathrm{~km} \mathrm{~s}^{-1}$. The line center velocities of the ${ }^{12} \mathrm{CO}$ emission from each pointed observation range from $V_{\mathrm{LSR}}=$ $5.1-6.1 \mathrm{~km} \mathrm{~s}^{-1}$, with a mean value of $5.6 \mathrm{~km} \mathrm{~s}^{-1}$. For a given position in the map, the error in the measurement of the line center is $\pm 0.3 \mathrm{~km} \mathrm{~s}^{-1}$. The $12 \mathrm{~m}$ data provide considerably better velocity resolution $\left(0.078\right.$ versus $\left.0.65 \mathrm{~km} \mathrm{~s}^{-1}\right)$ and the error in line center velocity is typically less than $\pm 0.10 \mathrm{~km} \mathrm{~s}^{-1}$. The widths of the ${ }^{12} \mathrm{CO}$ emission lines range from $\Delta V=0.6$ to
$1.5 \mathrm{~km} \mathrm{~s}^{-1}$ with a mean of $1.1 \mathrm{~km} \mathrm{~s}^{-1}$ in the $12 \mathrm{~m}$ observations. Figure 5 presents channel-by-channel maps of the data obtained with the $5 \mathrm{~m}$ MWO telescope. The $5 \mathrm{~m}$ data show that the three observed "clumps" are separated in velocity. The mean line center velocity of clump A differs by $0.9 \mathrm{~km} \mathrm{~s}^{-1}$ from the mean line center velocities of clumps $B$ and $C$. The $5 \mathrm{~m}$ data also show that there is a velocity gradient of 0.5 to $1 \mathrm{~m} \mathrm{~s}^{-1}$ across the cloud along a line from the SW boundary to the NE edge. On smaller spatial scales this global gradient is hidden by local variations in the line center velocity.

We can also examine the agreement between line center velocities for the various molecular species observed toward the star. Table 1 shows the line center velocities at the stellar position $(0,0)$ from both radio and optical data. Within the mutual uncertainties, all of the velocities are in harmony, which implies that all of the observed lines originate in the same cloud.

The ${ }^{13} \mathrm{CO}$ emission, observed along selected lines of sight through the cloud, is detected at the positions of strongest ${ }^{12} \mathrm{CO}$ emission and maps the same distribution of the molecular gas. However, along at least two lines of sight the ${ }^{13} \mathrm{CO}$ line profiles are more irregular (i.e., non-Gaussian) than the corresponding ${ }^{12} \mathrm{CO}$ line profiles. These two cases are shown in Figure 6. The ${ }^{13} \mathrm{CO}$ emission lines are narrower, but interestingly are not symmetric. These spectra provide weak evidence of multiple velocity components along the line of sight, 


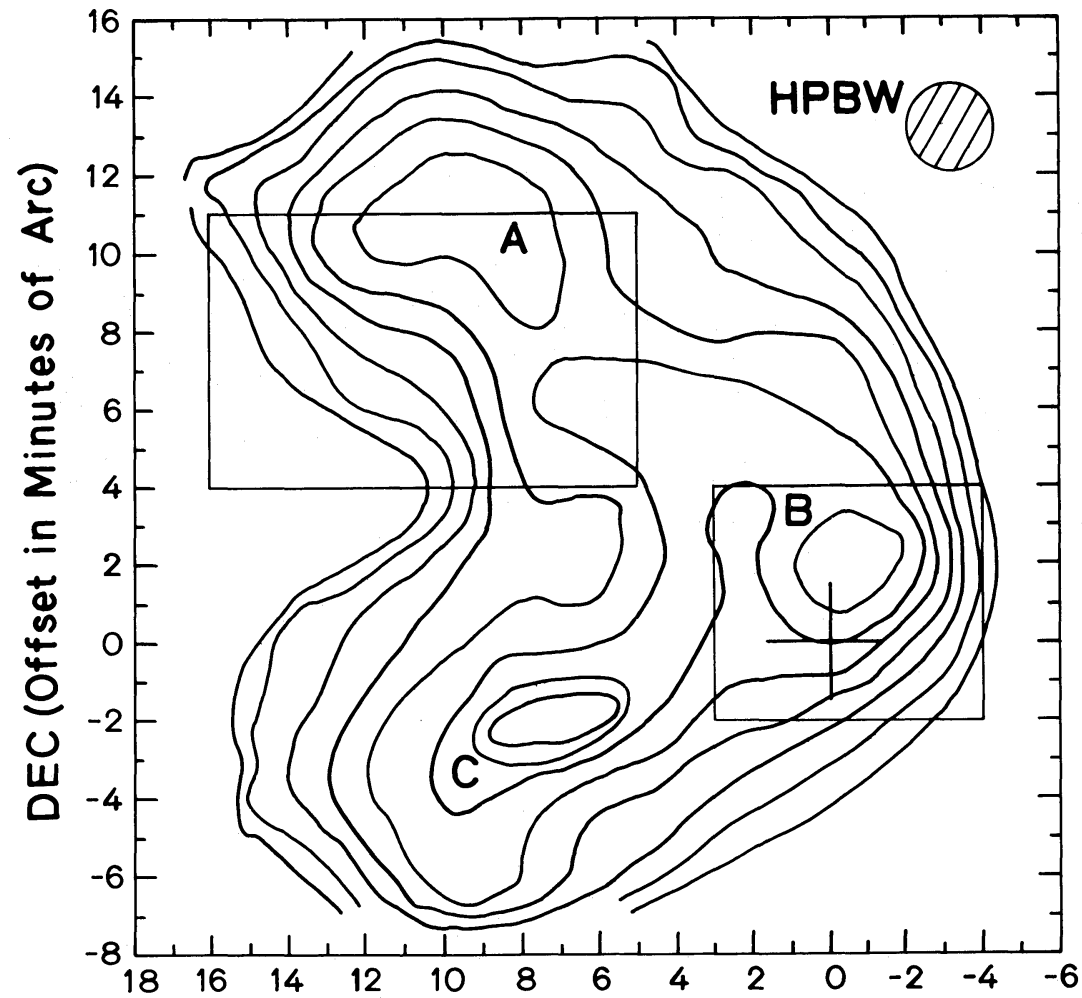

RA (Offset in Minutes of Arc)

FIG. 2.-Map of the peak $T_{R}^{*}$ observed at each pointed observation made with the MWO $5 \mathrm{~m}$ telescope. The telescope beam (HPBW) is 2.3 . Observations were spaced at $2^{\prime}$ intervals. Open contours indicate holes in the coverage of the observations. The plus sign indicates the position of HD 169454. The letters A, B, and C on the map indicate three regions of emission referred to in the text as "clumps." The lowest contour marks a peak $T_{R}^{*}$ of $1.0 \mathrm{~K}$. Each additional contour indicates an increase of $0.5 \mathrm{~K}$. The axes display the offset in arcminutes from the position of the star HD 169454. The box indicates the region mapped at higher angular resolution with the NRAO $12 \mathrm{~m}$ telescope.

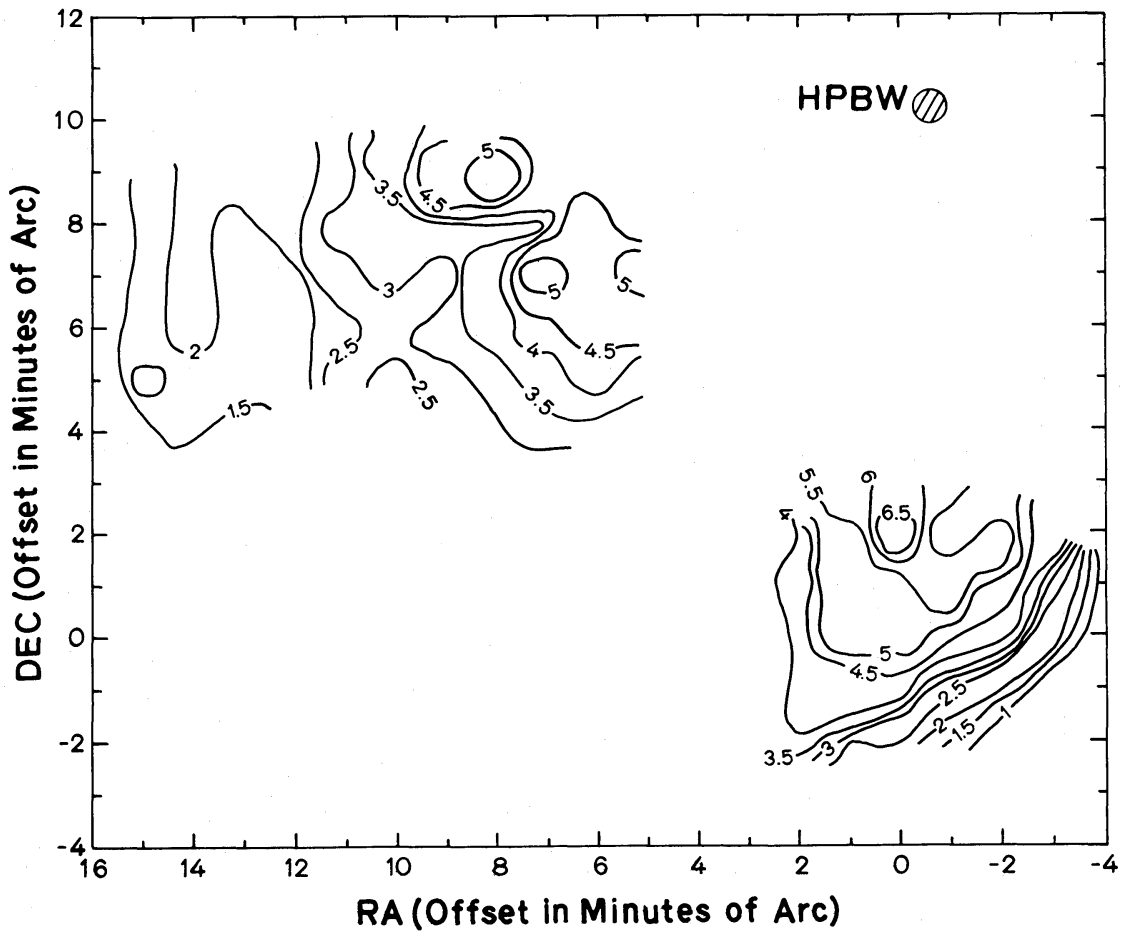

FIG. 3.-Map of the ${ }^{12} \mathrm{CO} J=1 \rightarrow 0$ integrated intensity made with the NRAO $12 \mathrm{~m}$ on Kitt Peak with a beam (HPBW) of $1^{\prime}$. A complete map was not obtained. Contours are spaced at intervals of $0.5 \mathrm{~K} \mathrm{~km} \mathrm{~s}^{-1}$. Observations were spaced at 1 minute intervals and covered the area indicated. The axes display the offset in arcminutes from the position of the star HD 169454. 


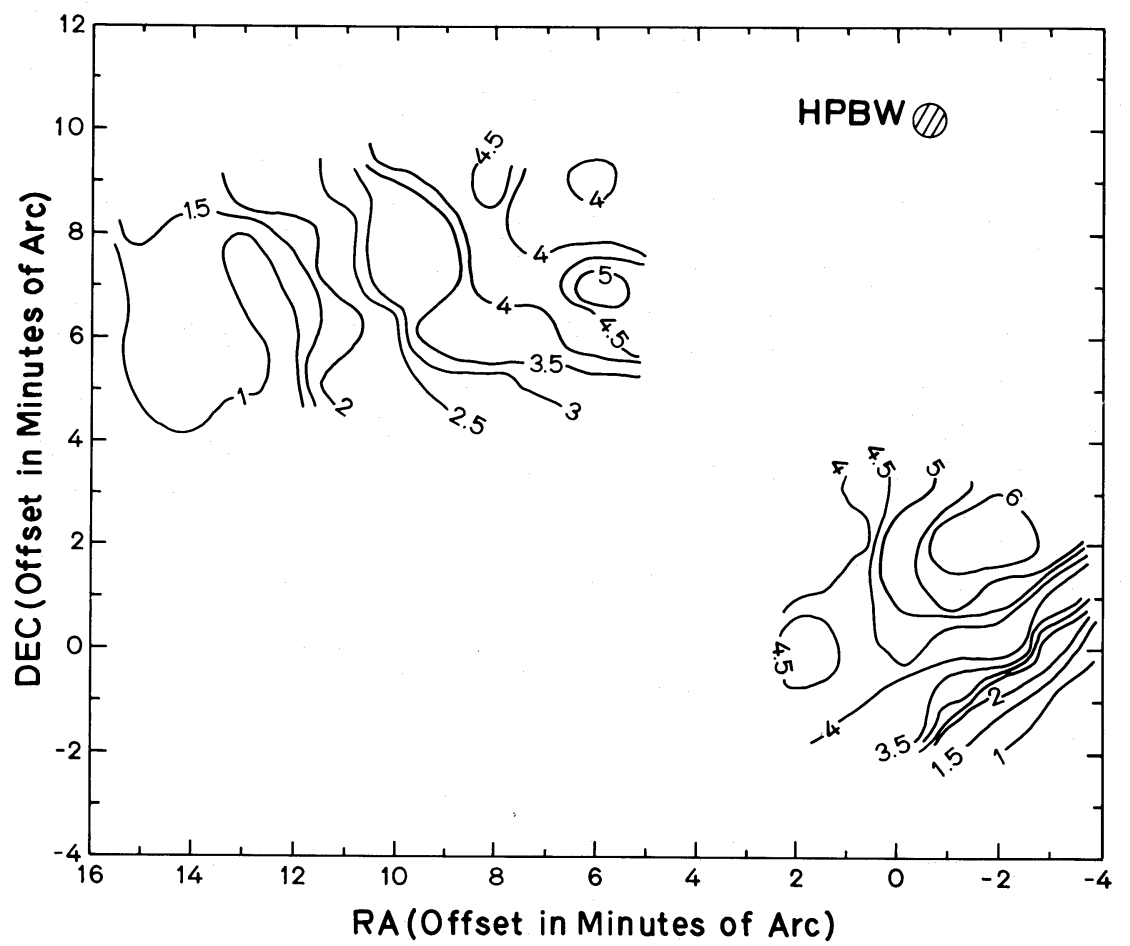

Fig. 4.-Map of the measured peak $T_{R}^{*}$ in the ${ }^{12} \mathrm{CO} J=1 \rightarrow 0$ line observed with the NRAO $12 \mathrm{~m}$ on Kitt Peak with a beam (HPBW) of $1^{\prime}$. A complete map was not obtained. Contours are spaced at intervals of $0.5 \mathrm{~K}$. Observations were spaced at $1^{\prime}$ intervals and covered the area indicated. The axes display the offset in arcminutes from the position of the star HD 169454.

which are separated by $0.5 \mathrm{~km} \mathrm{~s}^{-1}$, or less. Such small-scale velocity structure has been found in the $\mathrm{CO}$ emission from the diffuse cloud toward $\zeta$ Oph by Langer, Glassgold, and Wilson (1987), but our signal-to-noise ratio and resolution are not quite high enough to quantify similar structures in the cloud toward HD 169454. In all cases, the widths of the ${ }^{13} \mathrm{CO}$ lines are significantly smaller than those of the corresponding ${ }^{12} \mathrm{CO}$ lines. As the models of $\S$ IV suggest, ${ }^{13} \mathrm{CO}$ in such a relatively small, thin cloud is likely to be detectable over a substantially smaller volume than ${ }^{12} \mathrm{CO}$, so that the more abundant species samples a broader or qualitatively different velocity distribution.

\section{d) Derived Properties}

The determination from millimeter emission line data of $\mathrm{CO}$ column densities and of total molecular densities and masses is susceptible to problems. In the present case, the density implied by the rotational excitation of $\mathrm{C}_{2}$-although uncertain-is low enough, $n\left(\mathrm{H}_{2}\right) \approx 300 \mathrm{~cm}^{-3}$, that the $\mathrm{CO}$ might not be fully thermalized. Thus we compare in some detail the results of several different techniques for interpreting the $\mathrm{CO}$ emission.

The simplest approach to estimating the molecular content of a cloud is to apply a mean conversion factor, $\alpha=N\left(\mathrm{H}_{2}\right) / I_{\mathrm{CO}}$, which relates the integrated intensity of a $\mathrm{CO}$ emission line, $I_{\mathrm{Co}}=\int T_{R}^{*}(V) d V \mathrm{~K} \mathrm{~km} \mathrm{~s}^{-1}$, to the $\mathrm{H}_{2}$ column density. The use of such conversion factors has been reviewed by van Dishoeck and Black (1987), Kutner and Leung (1985), Israel (1985), and Liszt (1982), and specific issues in their use and calibration have been addressed by Bloemen et al. (1986) and Maloney and Black (1988). As a general comment, conversion factors, which are derived as mean properties for some sample of clouds (e.g., Dickman 1978; Martin and Barrett 1978; Frerking, Langer, and Wilson 1982), must be applied with caution to individual clouds.

Based on the global conversion factor $\alpha=2.5 \times 10^{20} \mathrm{~cm}^{-2}$ $\left(\mathrm{K} \mathrm{km} \mathrm{s}^{-1}\right)^{-1}$ obtained from $\gamma$-ray, $\mathrm{CO}$, and $\mathrm{H}$ I observations of the inner Galaxy and the Orion complex (Lebrun et al. 1983; Bloemen et al. 1984, 1986), the ${ }^{12} \mathrm{CO}$ data at positions $(0,0)$ and $(-2,2)$ imply $N\left(\mathrm{H}_{2}\right)=(1.4-1.6) \times 10^{21} \mathrm{~cm}^{-2}$. This factor has been applied to a sample of small, high-latitude molecular clouds by Magnani, Blitz, and Mundy (1985). A slightly larger value of $\alpha=3.0 \times 10^{20} \mathrm{~cm}^{-2}\left(\mathrm{~K} \mathrm{~km} \mathrm{~s}^{-1}\right)^{-1}$ is also often used in the literature (Young and Scoville 1982) and has been applied to a sample of southern high-latitude clouds (Keto and Myers 1986). Use of a conversion factor $\alpha_{13}=1 \times 10^{21} \mathrm{~cm}^{-2}$ $\left(\mathrm{K} \mathrm{km} \mathrm{s}^{-1}\right)^{-1}$, based on ${ }^{13} \mathrm{CO}$ measurements (cf. Kutner and Leung 1985) indicates $N\left(\mathrm{H}_{2}\right) \approx 1 \times 10^{21} \mathrm{~cm}^{-2}$ for the data at both positions $(0,0)$ and $(-2,2)$. A recent comparison of $\mathrm{CO}$ observations and far-infrared measurements of a high-latitude molecular cloud complex suggests a rather smaller conversion factor $\alpha=0.5 \times 10^{20} \mathrm{~cm}^{-2}\left(\mathrm{~K} \mathrm{~km} \mathrm{~s}^{-1}\right)^{-1}$ (de Vries, Heithausen, and Thaddeus 1987). If our cloud of modest extinction is considered to be more like the small, high-latitude clouds studied by de Vries et al. than like typical GMCs or dark clouds, then application of their conversion factor would imply $N\left(\mathrm{H}_{2}\right) \approx 3.3 \times 10^{20} \mathrm{~cm}^{-2}$.

Another conventional technique for determining $\mathrm{H}_{2}$ column densities from $\mathrm{CO}$ observations is to use the empirical relation

$$
N\left(\mathrm{H}_{2}\right)=(5.0 \pm 2.5) \times 10^{5} N_{\mathrm{LTE}}\left({ }^{13} \mathrm{CO}\right) \mathrm{cm}^{-2}
$$

derived for a sample of dark clouds by Dickman (1978). Here, the column density, $N_{\mathrm{LTE}}\left({ }^{13} \mathrm{CO}\right)$, of ${ }^{13} \mathrm{CO}$ in local thermodynamical equilibrium (LTE) is calculated according to the following assumptions: $(a)$ the ${ }^{12} \mathrm{CO}$ is fully thermalized so that the peak brightness temperature of the ${ }^{12} \mathrm{CO} \mathrm{J}=1 \rightarrow 0$ line is 


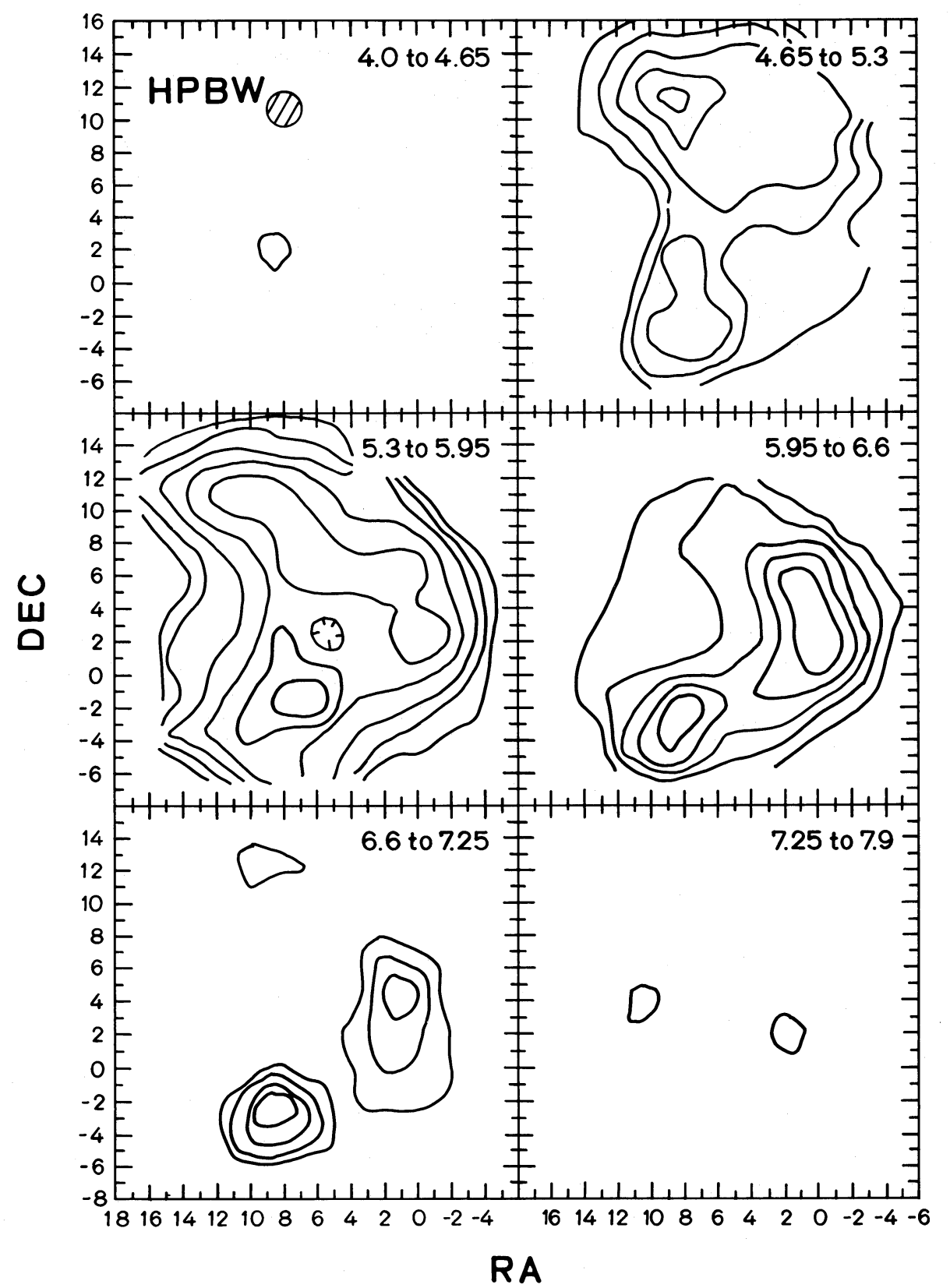

FIG. 5.-Individual maps of the ${ }^{12} \mathrm{CO} J=1 \rightarrow 0$ emission observed in a single channel of the $250 \mathrm{kHz}$ filterbank of the MWO $5 \mathrm{~m}$ telescope. Each channel is 0.65 $\mathrm{km} \mathrm{s}^{-1}$ in width. The velocity range included is indicated in the upper right-hand corner of each map. The axes indicate the offset in arcminutes from the position of the star HD 169454 . There is an interval of $0.3 \mathrm{~K} \mathrm{~km} \mathrm{~s}^{-1}$ between each contour. The lowest contour in the 4.65 to $5.3,5.3$ to 5.95 , and 5.95 to $6.6 \mathrm{maps}$ is $0.3 \mathrm{~K} \mathrm{~km}$ $\mathrm{s}^{-1}$. In the 4.0 to $4.65,6.6$ to 7.25 , and 7.25 to 7.9 maps the first contour is $0.1 \mathrm{~K} \mathrm{~km} \mathrm{~s}^{-1}$, the second $0.3 \mathrm{~K} \mathrm{~km} \mathrm{~s}^{-1}$, the third $0.6 \mathrm{~K} \mathrm{~km} \mathrm{~s} \mathrm{~s}^{-1}$, and the fourth $0.9 \mathrm{~K} \mathrm{~km}$ $s^{-1}$.

a measure of its excitation temperature, and $(b)$ the ${ }^{13} \mathrm{CO}$ is fully thermalized at the same excitation temperature (cf. Penzias, Jefferts, and Wilson 1971; Dickman 1978). At position $(0,0)$, this implies that $T_{\mathrm{ex}}\left({ }^{13} \mathrm{CO}\right)=T_{b}\left({ }^{12} \mathrm{CO}\right)=T=8.0 \mathrm{~K}$, that $N_{\mathrm{LTE}}\left({ }^{13} \mathrm{CO}\right)=1.2 \times 10^{15} \mathrm{~cm}^{-2}$, and that $N\left(\mathrm{H}_{2}\right)=6.0$ $\times 10^{20} \mathrm{~cm}^{-2}$. As discussed below, there is reason to believe that ${ }^{13} \mathrm{CO}$ in this cloud is subthermally excited (i.e., $T_{\mathrm{ex}}<T$ ) and that the true abundance ratio $N\left({ }^{13} \mathrm{CO}\right) / N\left(\mathrm{H}_{2}\right)$ is substantially smaller than Dickman's value for dark clouds.

In diffuse clouds that contain measured $\mathrm{H}$ and $\mathrm{H}_{2}$ (Savage $e t$ al. 1977), the mean relation between total column density and visual extinction is $N_{\mathrm{H}}=N(\mathrm{H})+2 N\left(\mathrm{H}_{2}\right)=1.59 \times 10^{21} A_{V}$. If the extinction toward HD 169454 were normal and if the abundances were comparable to those in diffuse clouds where $A_{V} \lesssim$ $1 \mathrm{mag}$, then the observed $E(B-V)=1.14 \mathrm{mag}$ would correspond to $N_{\mathrm{H}}=5.6 \times 10^{21} \mathrm{~cm}^{-2}$ or $N\left(\mathrm{H}_{2}\right) \leq 2.8 \times 10^{21} \mathrm{~cm}^{-2}$. The effects of the abnormal extinction on inferred gas column densities are difficult to predict. The signal level of the ultraviolet spectra of Seab, Snow, and Joseph (1981) is too low at the shortest wavelengths to provide an accurate measurement of the $\mathrm{H}$ I Ly $\alpha$ absorption and of $N(\mathrm{H})$. As discussed in $\S$ III $a$, Riegel and Crutcher (1972) have detected H $21 \mathrm{~cm}$ line selfabsorption in the direction of HD 169454, although with a relatively small optical depth $\tau \approx 0.2$ averaged over a $21^{\prime}$ beam. 

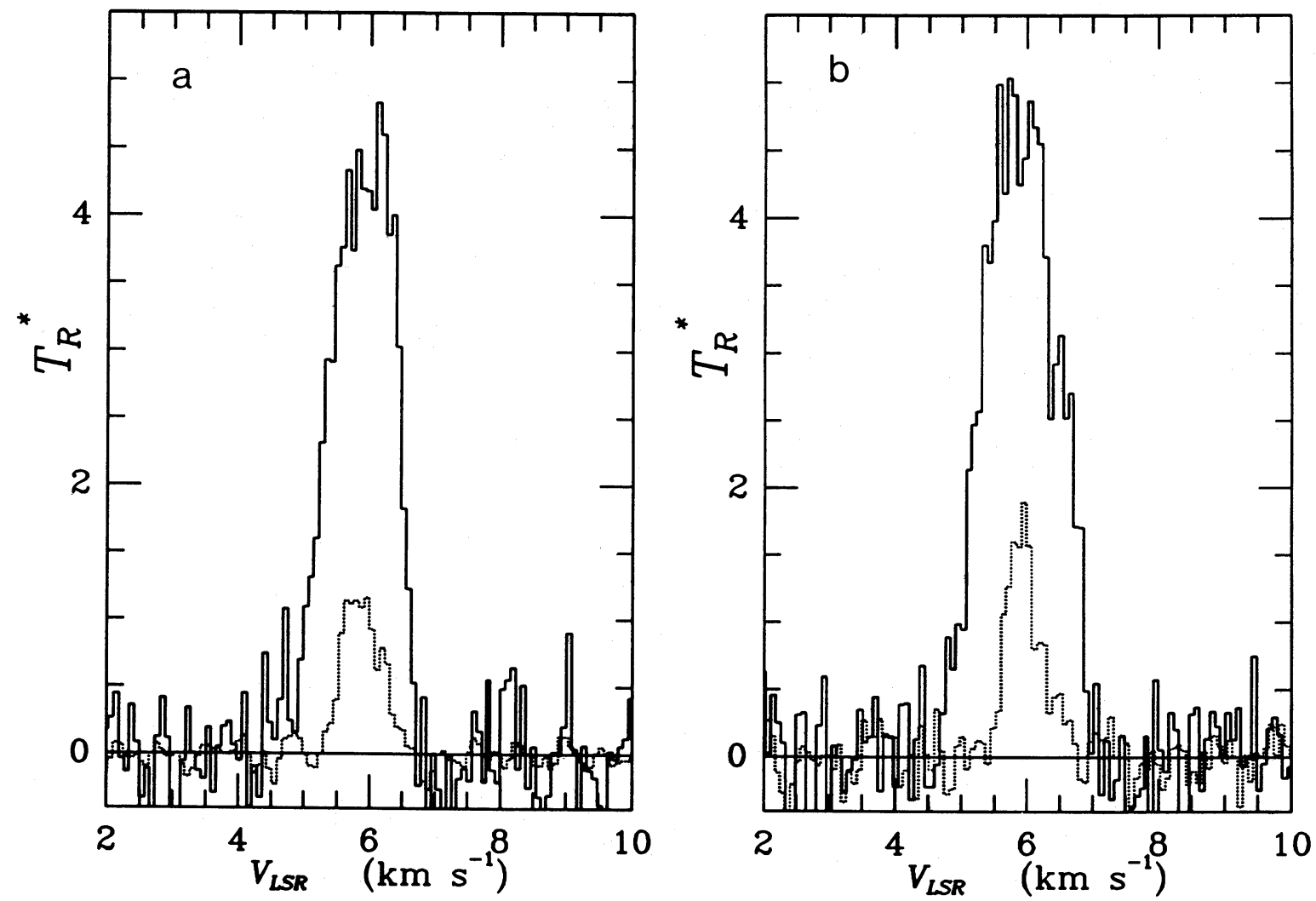

Fig. 6.-Spectra of the ${ }^{12} \mathrm{CO}$ and ${ }^{13} \mathrm{CO} J=1 \rightarrow 0$ emission $(a)$ toward the star and $(b)$ toward a position $0^{\prime} \mathrm{W} 2^{\prime} \mathrm{N}$ of the star. These spectra were obtained with the NRAO $12 \mathrm{~m}$ telescope using the $30 \mathrm{kHz}$ filter bank. Each channel is $0.078 \mathrm{~km} \mathrm{~s}^{-1}$ in width. The velocity axes refer to the local standard of rest $\left(V_{\mathrm{LSR}}\right)$. The ${ }^{12} \mathrm{CO}$ and ${ }^{13} \mathrm{CO}$ spectra are shown with solid and dotted lines, respectively.

In their investigation of the M16 region, Mufson et al. (1981) mapped the $H_{\text {I }} 21 \mathrm{~cm}$ line emission at 9.7 resolution over an area that includes the molecular cloud discussed here. From the data of Mufson et al. (1981) it is possible to estimate a 21 cm line optical depth at $V_{\mathrm{LSR}}=+5 \mathrm{~km} \mathrm{~s}^{-1}$ of $\tau_{21} \approx 2$ at the position of peak $\mathrm{B}$, considerably larger than that indicated by the maps of Riegel and Crutcher (1972). If the absorption is assumed to be very narrow (i.e., unresolved at the $1.67 \mathrm{~km} \mathrm{~s}^{-1}$ velocity resolution of the observations), then a column density $N(\mathrm{H})=1.82 \times 10^{18} \tau \Delta V T_{s} \gtrsim 9 \times 10^{19} \mathrm{~cm}^{-2}$ is inferred for a spin temperature $T_{s} \geq 15 \mathrm{~K}$. This limit is likely to be an underestimate of the atomic $\mathrm{H}$ owing to the low resolution of the 21 $\mathrm{cm}$ observations, and because the spin temperature of the broadly distributed $\mathrm{H}$ is expected to be higher than that of molecular material in the cloud core.

Column densities of several additional molecular species can be determined from the optical absorption line observations. For $\mathrm{C}_{2}$, a total column density $N\left(\mathrm{C}_{2}\right)=(7.3 \pm 1.4) \times 10^{13}$ $\mathrm{cm}^{-2}$ is found when a band oscillator strength $f_{20}=0.0010$ is used for the $\mathrm{A}^{1} \Pi_{u}-X^{1} \Sigma_{g}^{+}(2,0)$ transition. The column densities of $\mathrm{CN}$ are $N_{0}(\mathrm{CN})=(3.8 \pm 0.5) \times 10^{13}$ and $N_{1}(\mathrm{CN})=$ $(1.7 \pm 0.4) \times 10^{13} \mathrm{~cm}^{-2}$ in the $N=0$ and $N=1$ rotational states, respectively, based on a band oscillator strength of $f_{20}=7.6 \times 10^{-4}$ for the $\mathrm{A}^{2} \Pi-X^{2} \Sigma^{+}(2,0)$ transition. The rotational level populations in $\mathrm{CN}$ are consistent with an excitation temperature, $T_{\text {ex }}=2.9 \pm 0.4 \mathrm{~K}$, that is not significantly in excess of the cosmic background radiation temperature at the CN $N=1 \rightarrow 0$ transition frequency, $T_{\mathrm{bg}}=2.74 \pm 0.05 \mathrm{~K}$ (Crane et al. 1986). The $\mathrm{CN}$ and $\mathrm{C}_{2}$ lines are sufficiently weak that saturation effects do not complicate the analysis of column densities and excitation. The interstellar $\mathrm{CH}$ line, however, is relatively strong and partially saturated with an equivalent width $W_{\lambda}=24 \mathrm{~m} \AA$. If we assume that the $\mathrm{CH}$ absorption line is broadened by the same amount as the ${ }^{13} \mathrm{CO}$ $J=1 \rightarrow 0$ line in the direction of the star, corresponding to a Doppler parameter of $b=0.5 \mathrm{~km} \mathrm{~s}^{-1}$, then we find a column density $N(\mathrm{CH})=(4.6 \pm 0.8) \times 10^{13} \mathrm{~cm}^{-2}$.

The column density of $\mathrm{CH}$ is also observed to be strongly correlated with that of $\mathbf{H}_{2}$. For a sample of diffuse clouds, Danks, Federman, and Lambert (1984) found

$$
\log N\left(\mathrm{H}_{2}\right)=1.125 \log N(\mathrm{CH})+5.828 \mathrm{~cm}^{-2},
$$

while Mattila (1986) extended the relation to dark clouds, for which

$$
N\left(\mathrm{H}_{2}\right)=2.13 \times 10^{7} N(\mathrm{CH})+2.175 \times 10^{20} \mathrm{~cm}^{-2} .
$$

These relations imply $N\left(\mathrm{H}_{2}\right)=1.6 \times 10^{21}$ and $1.2 \times 10^{21}$ $\mathrm{cm}^{-2}$, respectively.

Column densities of $\mathrm{CO}$ and ${ }^{13} \mathrm{CO}$ can - in principle-be inferred directly from the observations. In order to interpret the observations it is necessary to have estimates of the number density of $\mathrm{H}_{2}, n\left(\mathrm{H}_{2}\right)$, and the kinetic temperature $T$. As mentioned in $\S \mathrm{I}$, the $\mathrm{C}_{2}$ molecule is a good diagnostic of the kinetic temperature and density of the cloud. Based on the procedure of van Dishoeck and Black (1982), the kinetic temperature is found to be $T \approx 15_{-5}^{+10} \mathrm{~K}$ and the number density of collision partners (presumably molecular hydrogen), $n\left(\mathrm{H}_{2}\right) \approx 300 \mathrm{~cm}^{-3}$. The density may be subject to an uncertainty of the order of a factor of 2. For the purpose of estimating column densities, we 
consider specifically our $12 \mathrm{~m}$ measurements of ${ }^{12} \mathrm{CO}$ and ${ }^{13} \mathrm{CO} J=1 \rightarrow 0$ lines together with the $5 \mathrm{~m}$ observations of ${ }^{12} \mathrm{CO}$ and ${ }^{13} \mathrm{CO} J=2 \rightarrow 1$ obtained by Lada and Blitz (1987). The beam sizes are comparable and both sets of data refer to the stellar position $(\Delta \alpha, \Delta \delta)=(0,0)$. Although there is good reason to doubt that the excitation (especially of ${ }^{13} \mathrm{CO}$ ) closely approaches a uniform thermal distribution of rotational populations, such an analysis is instructive. We use the term "uniform thermal excitation" to refer to a Boltzmann distribution of populations at a specified excitation temperature $T_{e x}$ in order to emphasize the distinction between this and local thermodynamic equilibrium (LTE). Strictly speaking, LTE further presumes that the microscopic motions are characterized by the same value of temperature as the internal energy distribution; i.e., that kinetic temperature $T=T_{\mathrm{ex}}$. With the assumption of uniform thermal populations and a background brightness temperature $T_{\mathrm{bg}}=2.74 \mathrm{~K}$, independent of wavelength, the two ${ }^{12} \mathrm{CO}$ lines imply $N(\mathrm{CO}) \geq 3 \times 10^{16} \mathrm{~cm}^{-2}$ and an excitation temperature $T_{\mathrm{ex}}=8.0 \mathrm{~K}$. The two ${ }^{13} \mathrm{CO}$ lines yield $N\left({ }^{13} \mathrm{CO}\right)=5 \times 10^{14} \mathrm{~cm}^{-2}$ and a rather lower $T_{\mathrm{ex}}=4.3$ $\mathrm{K}$. The stated uncertainties in measurement and calibration allow no solution with a common value of $T_{\mathrm{ex}}$ for all four lines of both species. As is apparent from Table 2 , the conventional "LTE column density" of ${ }^{13} \mathrm{CO}$ derived from the $J=1 \rightarrow 0$ lines is inconsistent with the measured intensity of the ${ }^{13} \mathrm{CO}$ $J=2 \rightarrow 1$ transition.

We have also performed full nonthermal, statistical equilibrium calculations of the excitation of 10 level ${ }^{12} \mathrm{CO}$ and ${ }^{13} \mathrm{CO}$ molecules. The radiative transfer is treated in terms of mean escape probabilities for an assumed uniform, spherical cloud. All rotationally inelastic collisions with $\mathrm{H}_{2}$ are included and the rates of absorption and stimulated and spontaneous emission are evaluated self-consistently. In this case again, the background radiation is given by $T_{\mathrm{bg}}=2.74 \mathrm{~K}$, independent of wavelength. The $\mathrm{H}_{2}+\mathrm{CO}$ collisional excitation rate coefficients of Schinke et al. (1985) have been adopted and have been augmented for some higher transitions by those of Flower and Launay (1985). Adoption of the rates of Flower and Launay for all transitions changes most calculated antenna temperatures by a few percent or less. Excitation by atomic $\mathrm{H}$ has also been examined with the use of the collisional rates of Chu and Dalgarno (1975). The cloud models of $\S$ IV suggest that the $\mathrm{H} / \mathrm{H}_{2}$ density ratio is sufficiently low in the $\mathrm{CO}$ emitting region that $\mathrm{H}$-impact excitation affects the emergent antenna temperatures at a level of only a few percent or less. Excitation of $\mathrm{CO}$ by $\mathrm{H}$ atoms is intrinsically more efficient than by $\mathrm{H}_{2}$ molecules (Chu and Dalgarno 1975), and the effects of $\mathrm{H}$ will be important in some diffuse clouds where its abundance is relatively high. Results of the statistical equilibrium calculations are compared with observation and with the results of the uniform thermal calculations in Table 2 . If we take a kinetic temperature $T \approx 15 \mathrm{~K}$ and a density $n\left(\mathrm{H}_{2}\right) \approx 300 \mathrm{~cm}^{-3}$, as suggested by the observed excitation of $C_{2}$, we find that the $J=1 \rightarrow 0$ line data at position $(0,0)$ are best fit with column densities $N(\mathrm{CO})=3.3 \times 10^{16} \mathrm{~cm}^{-2}$ and $N\left({ }^{13} \mathrm{CO}\right)=2.0 \times 10^{15} \mathrm{~cm}^{-2}$. No low-temperature, statistical equilibrium model of uniform, low density can reproduce exactly both the $J=1 \rightarrow 0$ and $J=2 \rightarrow 1$ measurements. For example, at $T=15 \mathrm{~K}$ and $n\left(\mathrm{H}_{2}\right)=300 \mathrm{~cm}^{-3}$, the column density that fits the $J=1 \rightarrow 0$ emission in ${ }^{12} \mathrm{CO}$ underestimates the strength of $J=2 \rightarrow 1$ by a factor of 1.5. Although the combined errors of absolute calibration for data obtained by different observers at different telescopes might approach a factor of 1.5 , the problem might also be explained by clumpiness in temperature or density such that the dominant contribution to the $J=2 \rightarrow 1$ emission arises under slightly different conditions than the bulk of the $J=1 \rightarrow 0$ emission. Alternatively, deviations from the assumed spherical geometry may affect the excitation and line formation. At very high densities, $n\left(\mathrm{H}_{2}\right)=1750-8000 \mathrm{~cm}^{-3}$, all the measurements can be represented well by single, uniform models. However, the isotope ratios inferred from the highdensity models seem unrealistic: $N\left({ }^{12} \mathrm{CO}\right) / N\left({ }^{13} \mathrm{CO}\right) \approx 8-10$.

The high-density models would also be in conflict with the low density inferred from the $\mathrm{C}_{2}$ excitation. Although the observed $\mathrm{C}_{2}$ absorption samples the same line of sight as the $\mathrm{CO}$ emission, the angular extent on the sky of the $\mathrm{CO}$ measurements is of the order of $1^{\prime}$, while the corresponding angular extent for $\mathrm{C}_{2}$ is the angular diameter of the background star.

TABLE 2

COMPARISON OF ObSERVED aNd CALCUlated CO LiNe INTENSITIES

\begin{tabular}{|c|c|c|c|c|c|c|c|c|c|}
\hline Type of Result ${ }^{\mathbf{a}}$ & $\begin{array}{c}T \\
(\mathbf{K})\end{array}$ & $\begin{array}{l}n\left(\mathrm{H}_{2}\right) \\
\left(\mathrm{cm}^{-3}\right)\end{array}$ & $\begin{array}{l}N\left({ }^{12} \mathrm{CO}\right) \\
\left(\mathrm{cm}^{-2}\right)\end{array}$ & $\begin{array}{c}T_{R}(1 \rightarrow 0) \\
(\mathrm{K})\end{array}$ & $\begin{array}{c}T_{R}(2 \rightarrow 1) \\
(\mathrm{K})\end{array}$ & $\begin{array}{c}T_{R}(3 \rightarrow 2) \\
(\mathrm{K})\end{array}$ & $\begin{array}{c}N\left({ }^{13} \mathrm{CO}\right) \\
\left(\mathrm{cm}^{-2}\right)\end{array}$ & $\begin{array}{c}T_{R}(1 \rightarrow 0) \\
(\mathrm{K})\end{array}$ & $\begin{array}{c}T_{R}(2 \rightarrow 1) \\
(\mathrm{K})\end{array}$ \\
\hline Observation $(0,0) \ldots \ldots \ldots$. & & $\ldots$ & & $4.7 \pm 0.1$ & $3.5 \pm 0.3^{b}$ & & & $1.2 \pm 0.09$ & $0.7 \pm 0.2^{b}$ \\
\hline 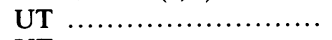 & 8.0 & $\ldots$ & $4.3(16)^{\mathrm{c}}$ & $4.70^{-}$ & 3.51 & 2.35 & $1.1(15)$ & 1.20 & 1.32 \\
\hline UT $\ldots \ldots \ldots \ldots \ldots \ldots \ldots \ldots \ldots$ & 4.3 & & $2.0(17)$ & 1.26 & 0.72 & 0.32 & $4.7(15)$ & 1.20 & 0.60 \\
\hline (n) & 10 & 300 & $9.0(16)$ & 4.78 & 2.36 & 0.482 & $3.0(15)$ & 1.26 & 0.452 \\
\hline SE $\ldots \ldots \ldots \ldots \ldots \ldots \ldots \ldots \ldots \ldots \ldots \ldots \ldots$ & 10 & 500 & $5.0(16)$ & 4.69 & 2.27 & 0.467 & $2.0(15)$ & 1.31 & 0.505 \\
\hline SE ….................... & 10 & 8000 & $9.3(15)$ & 4.75 & 3.51 & 1.21 & $9.0(14)$ & 1.27 & 1.38 \\
\hline SE $\ldots \ldots \ldots \ldots \ldots \ldots \ldots \ldots \ldots \ldots \ldots \ldots$ & 15 & 300 & $3.3(16)$ & 4.78 & 2.19 & 0.511 & $2.0(15)$ & 1.33 & 0.561 \\
\hline SE $\ldots \ldots \ldots \ldots \ldots \ldots \ldots \ldots \ldots \ldots \ldots \ldots \ldots \ldots$ & 20 & 300 & $2.2(16)$ & 4.80 & 2.21 & 0.595 & $1.5(15)$ & 1.26 & 0.588 \\
\hline SE ….................... & 20 & 500 & $1.25(16)$ & 4.75 & 2.20 & 0.607 & $1.1(15)$ & 1.28 & 0.687 \\
\hline SE …..................... & 20 & 2200 & $5.9(15)$ & 4.75 & 3.49 & 1.27 & $8.0(14)$ & 1.32 & 1.42 \\
\hline SE & 25 & 300 & $1.7(16)$ & 4.76 & 2.22 & 0.651 & $1.3(15)$ & 1.24 & 0.623 \\
\hline SE …....................... & 25 & 500 & $1.0(16)$ & 4.68 & 2.27 & 0.680 & $9.3(14)$ & 1.20 & 0.706 \\
\hline SE & 25 & 1750 & $5.6(15)$ & 4.70 & 3.50 & 1.35 & $7.2(14)$ & 1.21 & 1.32 \\
\hline
\end{tabular}

a UT stands for uniform thermal excitation with a Boltzmann population distribution at $T_{\mathrm{ex}}=T$. SE stands for statistical equilibrium with radiative transfer treated in terms of mean escape probabilities. Both types of calculations assume $T_{\mathrm{bg}}=2.74 \mathrm{~K}$. Comparison with observation assumes that $T_{R}^{*} \approx T_{R}$.

${ }_{\mathrm{b}}$ Observations of Lada and Blitz 1987

c At this excitation temperature, the observed values of $T_{R}$ are obtained for the ${ }^{12} \mathrm{CO} J=1 \rightarrow 0$ and $J=2 \rightarrow 1$ lines for any value of column density, $N(\mathrm{CO}) \geq 3 \times 10^{16} \mathrm{~cm}^{-2}$. 
This angular diameter is estimated to be

$$
\theta=4.4 \times 10^{-4}\left(\frac{R_{*}}{78 R_{\odot}}\right)\left(\frac{1660 \mathrm{pc}}{D_{*}}\right) \operatorname{arcsec},
$$

where $D_{*}$ is the distance to the star (see $\S \operatorname{III} a$ ) and $R_{*} \approx 78 R_{\odot}$ is its radius (Sterken and Wolf 1978). If there is extreme clumpiness on small scales, it is conceivable that there exist regions of very high density that dominate the $\mathrm{CO}$ emission but are not sampled by the bulk of the $\mathrm{C}_{2}$ along the line of sight to the star. Even so, we expect that most of the extended $\mathrm{CO}$ emission arises in gas similar to that probed by the $\mathrm{C}_{2}$. As indicated by entries in Table 2, the intensity of the $\operatorname{CO} J=3 \rightarrow 2$ line is predicted to be much stronger at high densities than at low density. Observations of this line would help to reveal the amount of high-density gas in the cloud. It is possible that clumpiness also affects the ${ }^{12} \mathrm{CO} /{ }^{13} \mathrm{CO}$ line ratios and the column density ratios inferred from them

Observations of ultraviolet or infrared absorption lines of interstellar CO toward HD 169454 would be extremely valuable. In this way, the total column density and the full rotational population distribution of $\mathrm{CO}$ could be determined accurately in a single set of measurements. HD 169454 may be somewhat too faint at $\lambda \approx 1500 \AA$ for observations at the highest resolution with the high resolution spectrograph on the Hubble Space Telescope, but the star is sufficiently bright in the $M$ band at $\lambda 4.8 \mu \mathrm{m}, M=3.35 \mathrm{mag}$ (Sneden et al. 1978), that high-resolution observations of the $(1,0)$ vibration-rotation band will be feasible. Specifically, for the statistical equilibrium calculation in Table 2 with $T=15 \mathrm{~K}, n\left(\mathrm{H}_{2}\right)=300 \mathrm{~cm}^{-3}$, and $N(\mathrm{CO})=3.3 \times 10^{16} \mathrm{~cm}^{-2}$, the predicted equivalent widths of the $(1,0) \mathbf{R}(0), \mathbf{R}(1)$, and $\mathbf{R}(2)$ lines are $0.285,0.285$, and $0.218 \AA$, respectively. These predictions include corrections for saturation with a Doppler parameter $b=0.5 \mathrm{~km} \mathrm{~s}^{-1}$. Absorption lines of this strength would be detectable at the $3 \sigma$ confidence level in spectra with signal-to-noise ratio of 15 at a resolving power of only $\lambda / \Delta \lambda=30000$.

On the basis of the existing observations, the column density of $\mathrm{CO}$ is not well determined. The data and two different forms of analysis allow $N(\mathrm{CO}) \approx(1-9) \times 10^{16} \mathrm{~cm}^{-2}$ and $N\left({ }^{13} \mathrm{CO}\right) /$ $N(\mathrm{CO}) \approx 0.03-0.1$. The upper limit on $\mathrm{H}_{2}$ column density, $N\left(\mathrm{H}_{2}\right) \leq 2.8 \times 10^{21} \mathrm{~cm}^{-2}$ based on a gas/extinction ratio typical of diffuse clouds, implies a linear scale $L \lesssim 3.0 \mathrm{pc}$ at $n\left(\mathrm{H}_{2}\right)=300 \mathrm{~cm}^{-3}$ and $L \lesssim 0.1 \mathrm{pc}$ at $n\left(\mathrm{H}_{2}\right)=8000 \mathrm{~cm}^{-3}$. As mentioned above, the overall size of the cloud is 4.4 (or 0.7 ) pc for a cloud distance $D=750$ (or 125) pc. Some structure is partly resolved on a scale of 0.2 (or 0.04 ) pc. If the cloud is at the nearer distance, the geometry appears to be more like that of a cylinder viewed end-on, provided that the density is low.

In summary, then, both global conversion factors for $\mathrm{CO}$ emission and the observed $\mathrm{CH}$ column density are consistent with a mean total column density $N\left(\mathrm{H}_{2}\right) \approx(1.0-2.0) \times 10^{21}$ $\mathrm{cm}^{-2}$ and with an upper limit $N\left(\mathrm{H}_{2}\right) \lesssim 2.8 \times 10^{21} \mathrm{~cm}^{-2}$ indicated by the poorly understood extinction toward the star. The column density of $\mathrm{CO}$ is not as well determined, but a value $N(\mathrm{CO}) \approx(2-9) \times 10^{16} \mathrm{~cm}^{-2}$ applies on the scale of the whole cloud. The conversion factor suggested by de Vries, Heithausen, and Thaddeus (1987) and the LTE column density derived by the method of Dickman (1978) apparently yield $\mathrm{H}_{2}$ column densities that are too low by factors of at least $2-5$. The agreement in total column densities derived from a global CO conversion factor and from the $\mathrm{CH}$ abundance and the extinction suggests that the small molecular cloud toward HD 169454 is thick enough so that its integrated $\mathrm{CO}$ emission and physical conditions are similar to those of the molecular clouds sampled by Bloemen et al. $(1984,1986)$.

\section{e) Mass Estimates}

Because mass estimates depend sensitively on the adopted distance, we will discuss the two cases separately.

\section{i) Large Distance}

The estimated column density $N\left(\mathrm{H}_{2}\right) \approx(1.0-2.0) \times 10^{21}$ $\mathrm{cm}^{-2}$ of the preceding section and the projected area of detectable CO emission, 330 square arcmin, imply a mass of molecular hydrogen

$$
M_{\mathrm{H}} \approx(250-500)\left(\frac{D}{750}\right)^{2} M_{\odot} .
$$

In more detail, the masses of the three main cloud components (labeled A, B, and C in Fig. 1) can be estimated individually. Using values of $I_{\mathrm{CO}}$ integrated over the angular areas of each of the clumps, and adopting a value of $\alpha=2.5 \times 10^{20} \mathrm{~cm}^{-2}(\mathrm{~K}$ $\left.\mathrm{km} \mathrm{s}^{-1}\right)^{-1}$, we find masses of 121,63 , and $75 M_{\odot}$ for features A, B, and C, respectively. As might be expected, the resulting sum of $260 M_{\odot}$ is slightly lower than the corresponding total mass estimated from a column density near the peak of emission and the total angular extent of the cloud. Note that this estimate does not include a contribution from atomic hydrogen. As will be shown in $\S \mathrm{IV}$, this contribution is expected to be small in relation to the uncertainties: approximately $10 \%-20 \%$ of the total $\mathrm{H}_{2}$ mass. However, when both the atomic hydrogen and the heavier elements are included, the total mass is approximately $40 \%-60 \%$ larger than that in $\mathrm{H}_{2}$ alone, based on the models of $\S$ IV.

The mass can also be estimated by a conventional "virial analysis" if the kinetic motions revealed by CO line widths are assumed to be balanced by the gravitational potential of the cloud. In this case, for a uniform velocity distribution, the mean ${ }^{12} \mathrm{CO}$ line width $\Delta V=1.1 \mathrm{~km} \mathrm{~s}^{-1}$ corresponds to a three-dimensional, mean-square velocity dispersion $\sigma=0.81$ $\mathrm{km} \mathrm{s}^{-1}$. The "virial mass" is thus

$$
M_{\mathrm{virial}} \approx \sigma^{2} \frac{d}{2 G} \approx 335\left(\frac{D}{750}\right) M_{\odot}
$$

based on an angular radius $\theta / 2=2.29 d(750 / D)$ arcmin $\approx 10$ arcmin for the cloud, where $d$ is its linear diameter in pc. The ratio $M_{\mathrm{H}} / M_{\text {virial }} \approx(0.75-1.5)(D / 750)$. When $M_{\mathrm{H}}$ is multiplied by a factor $1.4-1.6$ to correct for the mass of atomic hydrogen and heavy elements, $M_{\text {total }} / M_{\text {virial }} \approx(1.0-2.4)(D / 750)$; therefore, at distances $D \gtrsim 750 \mathrm{pc}$, the cloud would likely be virialized. Moreover, for any distance $300 \lesssim D \lesssim 1500 \mathrm{pc}, M_{\mathrm{H}}$ is still within a factor of 2 of the virial mass, which implies that for $D>300$ pc self-gravity is important in controlling the structure and evolution of this cloud. As a result, it is probable that for $D>300$ pc the cloud is bound and has a lifetime considerably longer than the crossing time $d / \sigma \approx 5.2 \times 10^{6}(D / 750) \mathrm{yr}$.

\section{ii) Small Distance}

If the molecular cloud is actually associated with the nearby cold atomic structure at $D \approx 125 \mathrm{pc}$, the mass estimates become $M_{\mathrm{H}} \approx 7-14 M_{\odot}$ and $M_{\text {virial }} \approx 60 M_{\odot}$. Even with correction for the atomic hydrogen and heavier elements, the implied mass falls far short of that required for virial equilibrium. The implied sound crossing time $d / \sigma \approx 9 \times 10^{5} \mathrm{yr}$ is 
sufficiently low that time-dependent effects on the chemistry may be expected. Gravity alone cannot maintain the persistent cloud structure. External pressures combined with anisotropic magnetic fields might account for the cloud and its geometry, if the smaller distance applies.

\section{THEORETICAL CLOUD MODELS}

In an empirical sense, the isolated molecular cloud at $V_{\mathrm{LSR}} \approx+6 \mathrm{~km} \mathrm{~s}^{-1}$ seen in the direction of HD 169454 can be described as a "small molecular cloud." It has a modest mass, a relatively small total column density, and a small central extinction in comparison with the properties of GMCs and dark molecular clouds. At the same time, it shows substructure like GMCs, but not necessarily on the same scale. From a theoretical perspective, this cloud can be considered to be a "translucent molecular cloud," with properties intermediate between those of the classical diffuse clouds and of opaque GMCs. The distinction is primarily one of thickness (or opacity to ultraviolet starlight), although translucent clouds might also tend to be somewhat denser than diffuse ones. The abundances of molecules other than $\mathrm{H}_{2}$ will be much higher in translucent clouds than in diffuse clouds owing to the reduced rates of photoprocesses inside the thicker clouds. Even so, photochemistry is expected to play a significant role throughout a translucent cloud, while it will be important only in the outermost layers of (or in the immediate vicinity of an embedded, hot star in) a GMC. The cloud toward HD 169454 appears to occupy a priviliged region of parameter space: its $\mathrm{CO}$ abundance is near the threshold where it can account for a large fraction of the available gas phase carbon. Detailed theoretical models of such clouds can be used to elucidate the connections between the theoretical notion of a "translucent cloud" and the empirical sobriquet of "small molecular cloud."

Detailed models have been computed using the techniques discussed by van Dishoeck and Black (1986) for diffuse clouds. Two major modifications have been made in those techniques. First, an elaborate description of the depth-dependent photodissociation of $\mathrm{CO}$ and of its isotopic varieties has been developed (van Dishoeck and Black 1988b). This incorporates the best spectroscopic data on dissociating transitions in the ultraviolet and treats explicitly the self-shielding in $\mathrm{CO}$ and the mutual shielding among the various isotopic varieties and $\mathrm{H}$ and $\mathrm{H}_{2}$. Second, the effects of all metals as abundant $\mathrm{as} \mathbf{R b}$ on the ionization balance have been incorporated into the chemical network (Lepp et al. 1988). Calculated column densities of selected atomic and molecular species are collected in Table 3 for two models of translucent clouds. These models are planeparallel structures and apply specifically to the line of sight to the star. The models are characterized principally by the central density $n_{\mathrm{H}}^{0}=n(\mathrm{H})+2 n\left(\mathrm{H}_{2}\right)$, the central kinetic temperature $T_{0}$, the scaling factor $I_{\mathrm{UV}}$ of the intensity of ultraviolet starlight relative to the adopted radiation field (Draine 1978), an efficiency factor for the formation of $\mathrm{H}_{2}$ on grain surfaces $y_{f}$, and a total column density of $\mathrm{H}_{2}$. In both models, $T$ and $n_{\mathrm{H}}$ respectively decrease and increase from edge to center in such a manner as to satisfy hydrostatic equilibrium. The basic structure of such models is governed by the depth-dependent abundance and excitation of $\mathrm{H}_{2}$ that are described in steady state. Abundances of more than 150 atoms, ions, and small molecules are evaluated in steady state at each depth step. See van Dishoeck and Black (1986) for details of the modeling techniques.
TABLE 3

Results of Cloud Models

\begin{tabular}{|c|c|c|c|}
\hline \multirow[b]{2}{*}{ PARAMETER } & \multicolumn{2}{|c|}{ MODEL $^{\mathbf{a}}$} & \multirow[b]{2}{*}{ OBSERVATIONS } \\
\hline & 1 & 2 & \\
\hline$n_{\mathrm{H}}^{0}\left(\mathrm{~cm}^{-3}\right) \ldots \ldots$ & 500 & 700 & \\
\hline 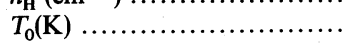 & 20 & 15 & $15_{-5}^{+10}$ \\
\hline 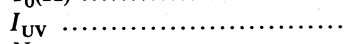 & 2 & 1 & $\ldots$ \\
\hline$N_{G} \ldots \ldots \ldots \ldots \ldots \ldots \ldots \ldots \ldots \ldots \ldots$ & 2 & 2 & $\cdots$ \\
\hline 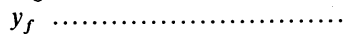 & 0.5 & 0.5 & $\ldots$ \\
\hline (n...................... & 0.1 & 0.1 & \\
\hline$A_{V}(\mathrm{mag}) \ldots \ldots$ & $2.25^{\mathrm{b}}$ & $2.95^{\mathrm{b}}$ & 3.5 \\
\hline$N\left(\mathrm{H}_{2}\right)\left(\mathrm{cm}^{-2}\right)$. & $1.5(21)$ & $2.2(21)$ & $\ldots$ \\
\hline$N(\mathrm{H})\left(\mathrm{cm}^{-2}\right) \ldots \ldots \ldots \ldots \ldots$ & $6.3(20)$ & $3.3(20)$ & $\ldots$ \\
\hline (Total mass) $/\left(\right.$ mass in $\left.\mathrm{H}_{2}\right)$ & 1.61 & 1.43 & $\ldots$ \\
\hline
\end{tabular}

Computed Column Densities $\left(\mathrm{cm}^{-2}\right)$

\begin{tabular}{|c|c|c|c|}
\hline C ... & $1.1(16)$ & $2.1(16)$ & $\ldots$ \\
\hline $\mathrm{C}^{+} .$. & $1.4(17)$ & $9.8(16)$ & \\
\hline${ }^{12} \mathrm{CO}$ & $2.0(16)$ & $1.0(17)$ & see Table 2 \\
\hline${ }^{13} \mathrm{CO} \ldots$ & $5.9(14)$ & $1.9(15)$ & see Table 2 \\
\hline $\mathrm{C}^{18} \mathrm{O} \ldots$ & $1.1(13)$ & $4.0(13)$ & $\ldots$ \\
\hline $\mathrm{OH} .$. & $3.5(14)$ & $2.1(15)$ & $\ldots$ \\
\hline OD ... & $8.0(11)$ & $1.6(13)$ & \\
\hline ......... & $4.7(13)$ & $7.6(13)$ & $4.6 \pm 0.8(13)$ \\
\hline${ }^{13} \mathrm{CH}$ & $1.0(12)$ & $2.4(12)$ & \\
\hline $\mathrm{C}_{2} \ldots$ & $9.9(13)$ & $1.6(14)$ & $7.3 \pm 1.4(13)$ \\
\hline (2) & $5.7(9)$ & $1.5(10)$ & \\
\hline $\mathrm{CN}$ & $1.4(13)$ & $4.9(13)$ & $5.6 \pm 0.9(13)$ \\
\hline $\mathrm{H}_{3}^{+} \ldots \ldots \ldots \ldots \ldots \ldots \ldots \ldots$ & $4.0(14)$ & $4.5(14)$ & $\ldots$ \\
\hline $\mathrm{H}_{2} \mathrm{D}^{+} \ldots$ & $3.6(12)$ & $6.4(12)$ & $\ldots$ \\
\hline $\mathrm{HCO}^{+} \ldots \ldots \ldots \ldots \ldots \ldots \ldots$ & $1.2(12)$ & $2.7(12)$ & $\ldots$ \\
\hline $\mathrm{CH}_{2}, \ldots \ldots \ldots$ & $2.2(14)$ & $4.0(14)$ & $\ldots$ \\
\hline $\mathrm{C}_{2} \mathrm{H}$ & $2.7(13)$ & $5.2(13)$ & $\ldots$ \\
\hline NH $\ldots \ldots \ldots+\ldots, \ldots \ldots \ldots$ & $1.5(11)$ & $7.3(11)$ & $\ldots$ \\
\hline $\mathrm{NH}_{2} \ldots \ldots \ldots \ldots \ldots \ldots \ldots \ldots$ & $3.7(11)$ & $1.9(12)$ & $\ldots$ \\
\hline NO & $1.6(12)$ & $5.7(13)$ & $\ldots$ \\
\hline HCN $, \ldots \ldots+\cdots, \ldots$ & $1.8(11)$ & $1.0(12)$ & $\ldots$ \\
\hline
\end{tabular}

\begin{tabular}{|c|c|c|c|}
\hline \multicolumn{4}{|c|}{ Central Densities $\left(\mathrm{cm}^{-3}\right)$ : } \\
\hline 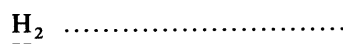 & 243.5 & 344.4 & $\ldots$ \\
\hline $\mathrm{H}$ & 13.0 & 11.2 & $\ldots$ \\
\hline Electrons ................... & $0.0184^{\circ}$ & $0.0157^{\mathrm{c}}$ & $\ldots$ \\
\hline
\end{tabular}

a See van Dishoeck and Black 1986 for definitions of symbols.

${ }^{b}$ Based on the possibly questionable assumption of average interstellar extinction; see text.

${ }^{c}$ Based on abundances for heavy elements other than carbon that are similar to those derived for the $\zeta$ Oph cloud by Lepp et al. 1988.

The two models of Table 3 have extinctions $A_{V}$ somewhat smaller than that observed toward HD 169454. This difference can be explained in several ways: $(a)$ part of the total extinction can be attributed to the more dilute interstellar medium along the rest of the line of sight to the star; $(b)$ the gas/extinction ratio in this cloud (cf. Seab, Snow, and Joseph 1981) may differ from the mean value in diffuse clouds adopted here ( $\$$ III $d) ;(c)$ if chemical equilibrium has not been fully attained, then the models may underestimate the amount of $\mathrm{H}$ and thus the total extinction in relation to the fixed column density of $\mathrm{H}_{2}$; and $(d)$ the assumed plane-parallel geometry may underestimate the amount of radiation penetrating the cloud and again the column density of $\mathrm{H}$ with respect to that of $\mathrm{H}_{2}$. The computed CO column densities bracket the range of values in Table 2 . The central densities and temperatures have been chosen to be consistent with those inferred from analysis of the $\mathrm{C}_{2}$ observations. The computed column densities of atomic hydrogen, $N(\mathrm{H})=6.2 \times 10^{20}$ and $3.3 \times 10^{20} \mathrm{~cm}^{-2}$ for models 1 and 2 , respectively, are in harmony with the lower limit inferred in 
$\S$ IIId. The models indicate spin temperatures for $\mathrm{H}$ of $T_{S} \gtrsim 40$ $\mathrm{K}$, so that the data of Mufson et al. (1981) suggest $N(\mathrm{H}) \gtrsim 2$ $\times 10^{20} \mathrm{~cm}^{-2}$, which is comparable to the theoretical values. Since the atomic hydrogen column density is sensitive to the value of $n_{\mathrm{H}}$, a high density, $n_{\mathrm{H}}>10^{3} \mathrm{~cm}^{-3}$, appears to be excluded. These considerations also suggest that only a small fraction of the mass of the cloud $10 \%-20 \%$, is in atomic form. The models include helium and heavier elements, which would increase the total mass by a factor 1.334 relative to that in atomic and molecular hydrogen alone. A fraction 0.0131 of the total mass is in the form of dust in these models. Table 3 lists the ratio of total mass to mass in the form of $\mathrm{H}_{2}$ : this correction factor for atomic hydrogen and heavy elements can be used to adjust the inferred molecular mass, $M_{\mathrm{H}}$, of $\S$ III $e$.

The column densities of $\mathrm{CH}$ and $\mathrm{C}_{2}$ in Table 3 are somewhat larger than the observed values, but are sensitive to the adopted gas-phase carbon abundance, to the branching ratios of some of the dissociative recombination reactions, to the adopted rate of radiative association $\mathrm{C}^{+}+\mathrm{H}_{2} \rightarrow \mathrm{CH}_{2}^{+}+h v$, and to the value of $I_{\mathrm{Uv}}$. The carbon abundance $[\mathrm{C}] /[\mathrm{H}]=4.7$ $\times 10^{-4} \delta_{\mathrm{C}}$ adopted here involves a fairly large depletion from the gas phase into solids, represented by a depletion factor $\delta_{\mathrm{C}}=0.1$. Computed column densities of $\mathrm{CN}$ are somewhat smaller than the observed value, but depend on uncertain rates of photodissociation and of reactions of $\mathrm{H}_{3}^{+}$with $\mathrm{N}$. A rather low rate coefficient of $10^{-12} \mathrm{~cm}^{3} \mathrm{~s}^{-1}$ was employed for the latter reaction. Predicted column densities of some other potentially observable species, such as $\mathrm{OH}$ and $\mathrm{H}_{3}^{+}$, are also listed. Central densities of $\mathrm{H}, \mathrm{H}_{2}$, and electrons are of interest for excitation calculations. The concentrations of most species are severely depth-dependent. In particular, the $\mathrm{CO}$ abundance is quite sensitive to the effects of shielding against ultraviolet starlight, while the $\mathrm{H}_{2}$ abundance is even more so, as illustrated in Figure 7. One important thing to note in Figure 7 is that the extent of the molecular cloud defined by the condition $n\left(\mathrm{H}_{2}\right) \geq n(\mathrm{H})$ is predicted to be significantly larger than that over which $\mathrm{CO}$ is present in detectable amounts that represent a large fraction of the total gas-phase carbon. This property is unlikely, however, to have a large effect on any of the mass estimates discussed above.

The fractionation of ${ }^{13} \mathrm{C}$ in $\mathrm{CO}$ is controlled by competing effects: ion-exchange reactions like

$$
{ }^{13} \mathrm{C}^{+}+{ }^{12} \mathrm{CO} \rightleftarrows{ }^{13} \mathrm{CO}+{ }^{12} \mathrm{C}^{+}
$$

lead to a relative enhancement of ${ }^{13} \mathrm{CO}$ at low temperature, while photodissociation of ${ }^{13} \mathrm{CO}$ tends to remain more rapid than that of ${ }^{12} \mathrm{CO}$ in translucent clouds. As a result, the ratio of column densities $N\left({ }^{12} \mathrm{CO}\right) / N\left({ }^{13} \mathrm{CO}\right)$ can be quite sensitive to details in regions where photodissociation dominates the destruction of one or both species. The photochemistry of $\mathrm{CO}$ will be described in greater detail elsewhere (van Dishoeck and Black 1988b). The models described here have been calculated with a low overall abundance ratio $\left[{ }^{12} \mathrm{C}\right] /\left[{ }^{13} \mathrm{C}\right]=45$ in all forms, as suggested for diffuse clouds (Hawkins and Jura 1987). As Tables 2 and 3 indicate, the observations of ${ }^{12} \mathrm{CO}$ and ${ }^{13} \mathrm{CO}$ can be reproduced by such models.

The models summarized in Tables 3 and 4 represent only two possible cases. Similar models with somewhat higher or lower densities, values of $I_{\mathrm{UV}}$, and total column densities could be constructed that represent the observations fairly well. The $\mathrm{CO}$ abundance is very sensitive to the value of $I_{\mathrm{UV}}$ but rather less so to $\delta_{\mathrm{C}}$. A small value of $\delta_{\mathrm{C}} \lesssim 0.1$ is apparently needed to account for the $\mathrm{CH}$ and $\mathrm{C}_{2}$, if the $\mathrm{C}^{+}+\mathrm{H}_{2}$ radiative association rate coefficient is as large as $7 \times 10^{-16} \mathrm{~cm}^{3} \mathrm{~s}^{-1}$, the value inferred from models of diffuse clouds by van Dishoeck and Black (1986). Substantial depletion of carbon is probably consistent with the abnormal grains seen in this direction (Seab, Snow, and Joseph 1981).

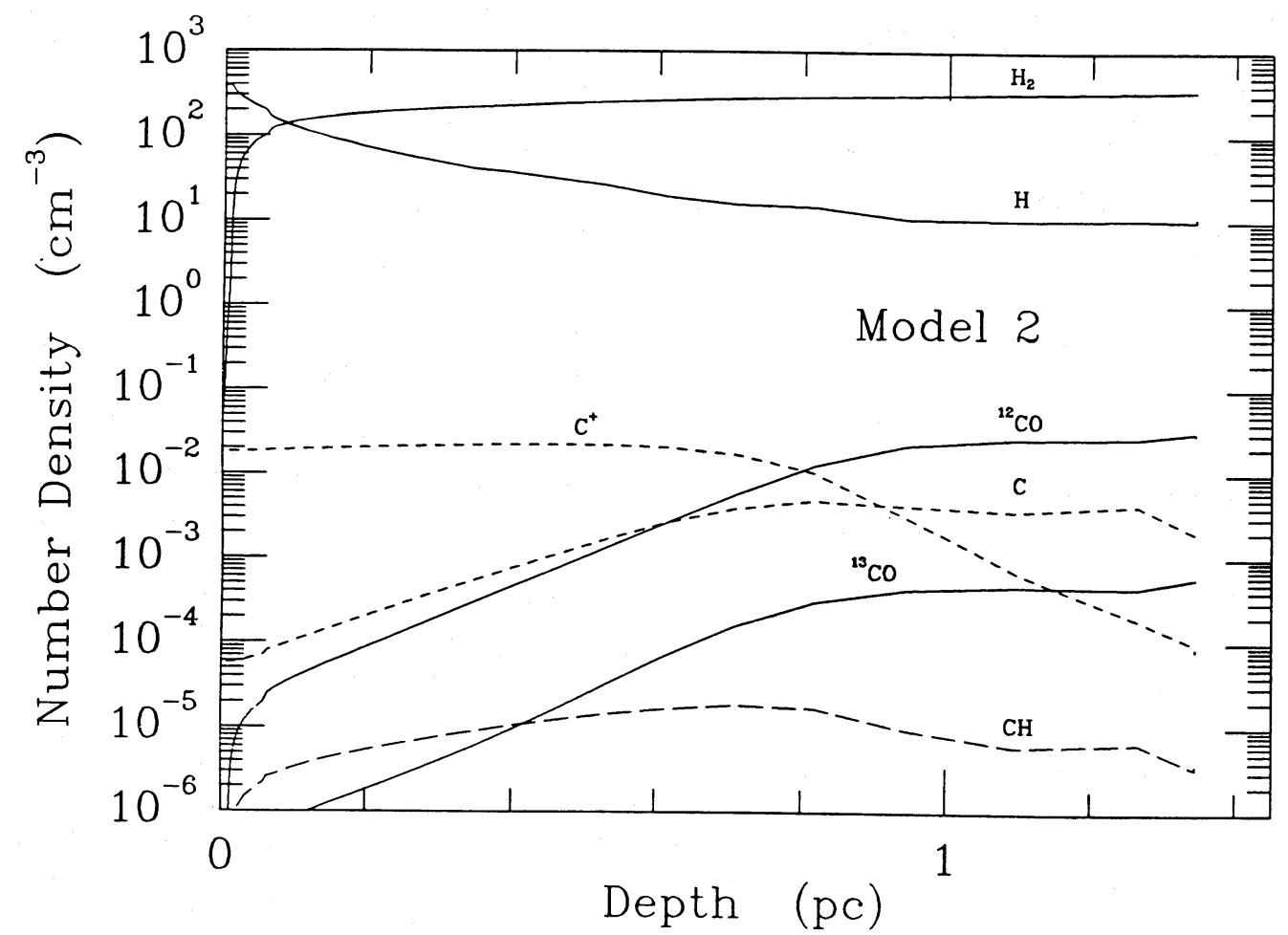

FIG. 7.-Depth-dependent number densities of major species in model 2 of the line of sight toward HD 169454. See Table 3 for the model properties. 
TABLE 4

Predicted Observable Properties

\begin{tabular}{|c|c|c|}
\hline Property & Model 1 & Model 2 \\
\hline $\mathrm{C}_{\mathrm{I}} \lambda 609 \mu \mathrm{m}, \mathrm{I}\left(\mathrm{ergs} \mathrm{s}^{-1} \mathrm{~cm}^{-2} \mathrm{sr}^{-1}\right)$ & $1.9(-7)$ & $3.3(-7)$ \\
\hline$C_{\text {I }} \lambda 370 \mu \mathrm{m}, \mathrm{I}\left(\mathrm{ergs} \mathrm{s}^{-1} \mathrm{~cm}^{-2} \mathrm{sr}^{-1}\right)$ & $1.3(-6)$ & $2.4(-6)$ \\
\hline$C_{\text {II }} \lambda 158 \mu \mathrm{m}, I\left(\mathrm{ergs} \mathrm{s}^{-1} \mathrm{~cm}^{-2} \mathrm{sr}^{-1}\right)$ & $2.2(-5)$ & $3.6(-5)$ \\
\hline $\mathrm{O}_{1} \lambda 63 \mu \mathrm{m}, \mathrm{I}\left(\mathrm{ergs} \mathrm{s}^{-1} \mathrm{~cm}^{-2} \mathrm{sr}^{-1}\right) .$. & $2.9(-7)$ & $4.9(-7)$ \\
\hline $\mathrm{O}_{\mathrm{I}} \lambda 145 \mu \mathrm{m}, \mathrm{I}\left(\mathrm{ergs} \mathrm{s}^{-1} \mathrm{~cm}^{-2} \mathrm{sr}^{-1}\right)$ & $1.2(-3)$ & $1.2(-3)$ \\
\hline$C_{\text {I }} 157 \alpha, T_{R} \Delta V\left(\mathrm{~K} \mathrm{~km} \mathrm{~s}^{-1}\right)^{\mathrm{a}} \ldots \ldots \ldots$ & $5.8(-4)$ & $2.6(-4)$ \\
\hline $\mathrm{CN}, N=1 \rightarrow 0, v=113.5 \mathrm{GHz}, T_{R}(\mathrm{~K})^{\mathrm{b}}$ & 0.019 & 0.018 \\
\hline $\mathrm{CN}, N=1 \rightarrow 0, v=113.2 \mathrm{GHz}, T_{R}(\mathrm{~K})^{\mathrm{b}}$ & 0.013 & 0.012 \\
\hline
\end{tabular}

${ }^{a}$ Non-LTE intensity based upon departure coefficients of Salem and Brocklehurst 1979.

${ }^{b} \mathrm{CN}$ rotational line intensities based upon a 15 state statistical equilibrium excitation calculation and the observed column density for an assumed $\Delta V=1.0 \mathrm{~km} \mathrm{~s}^{-1}$.

The models can also be used to predict other observable properties that could be applied as tests of the theory in the future. Predicted intensities of some emission and absorption lines are presented in Table 4 . The predicted intensities of the atomic fine-structure transitions are based upon a full treatment of the radiative and collisional processes that govern the fine-structure level populations at each depth step in the models and include allowance for stimulated emission and optical depth effects. The intensity of a carbon radio recombination line is also shown. Some of the atomic line intensities are very sensitive to the temperature structures in the outer parts of such clouds. The two models discussed here are rather similar in this respect. The peak radiation temperatures of the two spin components of the $N=1 \rightarrow 0$ rotational transition of $\mathrm{CN}$ are presented. These predictions combine the observed column density with a statistical equilibrium calculation in which the temperature and central densities of $\mathrm{H}_{2}$ and electrons in the models are adopted. In this treatment of a 15-state $\mathrm{CN}$ molecule, collisions with both $\mathrm{H}_{2}$ and electrons are included. The neutral impact cross sections are not known for $\mathrm{CN}$, so a downward cross section of $\sigma=5 \times 10^{-16} \mathrm{~cm}^{2}$ for $N=1 \rightarrow 0$ has been assumed, and the cross sections for other transitions have been assumed to scale according to the radiative transition probabilities. This yields collisional excitation rate coefficients very similar to those for CO and CS. The electron-impact rates have been computed by Allison and Dalgarno (1971). As in diffuse clouds, the electron fraction is large enough so that electron-impact excitation completely dominates the collisional effects on the rotational populations of $\mathrm{CN}$. Consideration of $\mathrm{H}_{2}$-impact excitation alone would lead to lines that are weaker by factors of 2.5-3. The computed excitation temperatures for $N=0$ and $1, T_{\mathrm{ex}}=2.8 \mathrm{~K}$ in both models, are consistent with the value determined from the absorption line measurements.

The empirical mass estimates discussed above for $D \approx 750$ pc support the assumption of hydrostatic equilibrium in the model clouds. If the cloud is nearer and smaller, equilibrium may also obtain if external pressure or magnetic fields support a stable structure. As described in van Dishoeck and Black (1986), such model clouds are finite in mass owing to an external boundary pressure $P_{1}$. For model 1 , the total external pressure (thermal plus turbulent) is $P_{1} / k=1.2 \times 10^{5} \mathrm{~cm}^{-3} \mathrm{~K}$, while the thermal component alone is a factor of 5 times smaller. The corresponding total boundary pressure in model 2 is $P_{1} / k=1.5 \times 10^{5} \mathrm{~cm}^{-3} \mathrm{~K}$. The questions of the stability of such clouds, which are characterized by polytropes of negative index, and of the relative importance of self-gravity and external pressure have been addressed by Viala and Horedt (1974), Dickman and Clemens (1983), and Maloney $(1987,1988)$.

Comparison of detailed models with observations shows that the cloud toward HD 169454 can be interpreted rather well. Predictions of additional observable properties will allow the theory to be tested and the interpretation refined.

\section{CONCLUSIONS}

The observed and derived properties of the small molecular cloud toward HD 169454 have been discussed. The CO emitting gas at $V_{\mathrm{LSR}} \approx 6 \mathrm{~km} \mathrm{~s}^{-1}$ is well isolated from background gas. Within the cloud three "clumps" are clearly observable and evidence exists of structure on increasingly smaller scales. Although the cloud is not uniform in its properties, we have shown that the evident complexity of the distribution and state of the molecular gas can begin to be understood when a combination of observational diagnostics are used to constrain detailed models. If the cloud is as close as $125 \mathrm{pc}$, then the estimated mass, $7-14 M_{\odot}$, is too small for virial equilibrium to hold. Only for distances $D>300 \mathrm{pc}$ are masses estimated by counting molecules and from application of the virial theorem comparable.

It is fortunate that the line of sight to HD 169454 passes almost through the peak of the $\mathrm{CO}$ emission in the cloud (clump B). As a result, diagnostic observations independent of $\mathrm{CO}$ emission can be made and analyzed. In conjunction with detailed theoretical models, it is possible to construct a fairly satisfying picture of the structure and physical conditions within this cloud. Further understanding of the nature and role of small molecular clouds as components of the interstellar medium will benefit from additional detailed studies of individual clouds.

We are very grateful to the referee, Richard Crutcher, for pointing out to us the important evidence that the cloud may be very nearby. B. T. J. would like to thank Larry Strom, Phil Jewell, Betty Stobie, Michael Margulis, and Dan Clemens for valuable discussions about the acquisition, calibration, and reduction of the millimeter data. J. H. B. and E. F. vD. are grateful to the Institute for Advanced Study for hospitality and for partial support through NSF grant PHY 86-20266. This work has been supported in part by NASA through grant NAGW-763 to the University of Arizona and by the NSF through grant RII 86-20342 to Princeton University.

\section{REFERENCES}

Allison, A. C., and Dalgarno, A. 1971, Astr. Ap., 13, 331.

Blitz, L. 1987, in Physical Processes in Interstellar Clouds, ed. G. E. Morfill and M. Scholer (Dordrecht: Reidel), p. 35.

Blitz, L., Magnani, L., and Mundy, L. 1984, A p. J. (Letters), 282, L9.

Bloemen, J. B. G. M., Caraveo, P. A., Hermsen, W., Lebrun, F., Maddalena,

R. J., Strong, A. W., and Thaddeus, P. 1984, Astr. Ap., 139, 37.

Bloemen, J. B. G. M., et al. 1986, Astr. Ap., 154, 25.

Breger, M. 1986, Ap. J., 309, 311.
Breger, M. 1987, Ap. J., 319, 754.

Chu, S.-I, and Dalgarno, A. 1975, Proc. Roy. Soc. A, 342, 191.

Clemens, D. P. 1985, Ap. J., 288, 422

Clemens, D. P., Sanders, D. B., Scoville, N. Z., and Solomon, P. M. 1986, Ap. J. Suppl., 60, 297.

Crane, P., Hegyi, D. J., Mandolesi, N., and Danks, A. C. 1986, Ap. J., 309, 822.

Crutcher, R. M. 1985, Ap. J., 288, 604.

Crutcher, R. M., and Chu, Y.-H. 1985, Ap. J., 290, 251. 
Crutcher, R. M., and Lien, D. J. 1984, in IAU Colloquium 81 The Local Interstellar Medium, ed. Y. Kondo, F. C. Bruhweiler, and B. D. Savage, (NASA CP-2345), p. 117

Danks, A. C., Federman, S. R., and Lambert, D. L. 1984, Astr. Ap., 130, 62.

de Vries, H. W., Heithausen, A., and Thaddeus, P. 1987, Ap. J., 319, 723.

Dickman, R. L. 1978, Ap. J. Suppl., 37, 407.

Dickman, R. L., and Clemens, D. P. 1983, Ap. J., 271, 143

Draine, B. T. 1978, Ap. J. Suppl., 36, 595.

Falgarone, E., and Pérault, M. 1987, in Physical Process in Interstellar Clouds,

ed. G. E. Morfill and M. Scholer (Dordrecht: Reidel), p. 59.

Federman, S. R., and Willson, R. F. 1984, Ap. J., 283, 626 .

Flower, D. R., and Launay, J. M. 1985, M.N.R.A.S., 214, 271

Frerking, M. A., Langer, W. D., and Wilson, R. W. 1982, Ap. J., 262, 590.

Gordon, K. J., and Arny, T. T. 1984, A.J., 89, 672.

Gredel, R., and Münch, G. 1986, Astr. Ap., 154, 336.

Gunn, J. E., Knapp, G. R., and Tremaine, S. D. 1979, A.J., 84, 1181

Guthrie, B. N. G. 1987, Quart. J.R.A.S., 28, 289.

Hawkins, I., and Jura, M. 1987, Ap. J., 317, 926.

Humphreys, R. M. 1978, Ap. J. Suppl., 38, 309.

Israel, F. P. 1985, in New Aspects of Galaxy Photometry, ed. J. L. Nieto (NY: Springer), p. 101.

Keto, E. R., and Myers, P. C. 1986, Ap. J., 304, 461.

Knapp, G. R., and Bowers, P. F. 1987, preprint.

Kutner, M. L., and Leung, C. M. 1985, Ap. J., 291, 188.

Kutner, M. L., and Ulich, B. L. 1981, Ap. J., 250, 350.

Lada, E. A., and Blitz, L. 1988, Ap. J. (Letters), 326, L69.

Langer, W. D., Glassgold, A. E., and Wilson, R. W. 1987, Ap. J., 322, 450.

Lebrun, F., et al. 1983, Ap.J., 274, 231.

Lepp, S., Dalgarno, A., van Dishoeck, E. F., and Black, J. H. 1988, Ap. J., 329, 418

Liszt, H. S. 1982, Ap. J., 262, 198.

Magnani, L., Blitz, L., and Mundy, L. 1985, Ap. J., 295, 402.

Magnani, L., Lada, E., and Blitz, L. 1986, A p. J., 301, 395.
Maloney, P. R. 1987, Ph.D thesis, University of Arizona.

Maloney, P. R. 1988, Ap. J., submitted.

Maloney, P. R., and Black, J. H. 1988, Ap. J., 325, 389.

Martin, R. N., and Barrett, A. H. 1978, Ap. J. Suppl., 36, 1.

Mattila, K. 1986, Astr. Ap., 160, 157.

Mufson, S. L., Fountain, W. F., Gary, G. A., Howard, W. F., III, O’Dell, C. R., and Wolf, M. T. 1982, Ap. J., 248, 992.

Penzias, A. A., and Burrus, C. A. 1973, Ann. Rev. Astr. Ap., 11, 51

Penzias, A. A., Jefferts, K. B., and Wilson, R. W. 1971, Ap. J., 165, 229.

Riegel, K. W., and Crutcher, R. M. 1972, Astr. Ap., 18, 55.

Salem, M., and Brocklehurst, M. 1979, Ap. J. Suppl., 39, 633.

Sanders, D. B., Clemens, D. P., Scoville, N. Z., and Solomon, P. M. 1986, Ap. J. Suppl., 60, 1 .

Savage, B. D., Bohlin, R. C., Drake, J. F., and Budich, W. 1977, Ap. J., 216, 291.

Schinke, R., Engel, V., Buck, U., Meyer, H., and Diercksen, G. H. F. 1985, $A p$. $J ., 299,939$.

Seab, C. G., Snow, T. P., Jr., and Joseph, C. L. 1981, Ap. J., 246, 788.

Sneden, C., Gehrz, R. D., Hackwell, J. A., York, D. G., and Snow, T. P. 1978, Ap. $J ., 223,168$.

Sterken, C., and Wolf, B. 1978, Astr. Ap., 70, 641

Ulich, B. L., and Haas, R. W. 1976, Ap. J. Suppl., 30, 247.

van Dishoeck, E. F., and Black, J. H. 1982, Ap.J., 258, 533

- 1984, ESO Messenger, 38, 16. 1986, Ap. J. Suppl., 62,109. 1987, in Physical Processes in Interstellar Clouds, ed. G. E. Morfill and M. Scholer (Dordrecht: Reidel), p. 241. $1988 a$, in preparation. $1988 b$, submitted to $A p . J$.

Viala, Y., and Horedt, Gp. 1974, Astr. Ap., 33, 195

Walborn, N. R. 1972, A.J., 77, 312.

White, R.E. 1984a, Ap. J., 284, 685

. 1984b, Ap. J., 284, 695 .

Young, J. S., and Scoville, N. Z. 1982, Ap. J., 258, 188

John H. Black, Buell T. Jannuzi, and Charles J. Lada: Steward Observatory, University of Arizona, Tucson, AZ 85721

Ewine F. van DishoeCK : Princeton University Observatory, Peyton Hall, Princeton, NJ 08544 